\title{
On the Gibbs Properties of Bernoulli Convolutions Related to $\beta$-Numeration in Multinacci Bases*
}

\author{
By \\ Eric Olivier $^{1, \dagger}$, Nikita Sidorov ${ }^{2, \downarrow}$, and Alain Thomas ${ }^{3}$ \\ ${ }^{1}$ Université de Provence, Marseille, France \\ ${ }^{2}$ The University of Manchester, UK \\ ${ }^{3}$ Centre de Mathématiques et d'Informatique, Marseille, France \\ Communicated by K. Schmidt
}

Received January 5, 2004; accepted in revised form November 17, 2004 Published online April 6, 2005 (C) Springer-Verlag 2005

\begin{abstract}
We consider infinitely convolved Bernoulli measures (or simply Bernoulli convolutions) related to the $\beta$-numeration. A matrix decomposition of these measures is obtained in the case when $\beta$ is a PV number. We also determine their Gibbs properties for $\beta$ being a multinacci number, which makes the multifractal analysis of the corresponding Bernoulli convolution possible.
\end{abstract}

2000 Mathematics Subject Classification: 28A12, 11A67, 15A48

Key words: Weak Gibbs measure, Bernoulli convolution, $\beta$-numeration, PV number, continued fraction, infinite matrix product

\section{Introduction}

The Bernoulli convolutions have been studied since the early 1930's $[12,5,13,8]$ and more recently, from the 1990's onward, following the work of Alexander and Yorke [1]. They have also been considered in view of their applications to fractal geometry [19, 18, 16, 27, 14] and ergodic theory [30, 29] (see [23] for further details and references). Our approach is motivated by the fact that the Bernoulli convolution associated with the golden ratio (usually called the Erdoss measure) proves to be weak Gibbs [7] and, thus, satisfies the multifractal formalism. In the present paper we aim to generalize this result.

We focus our attention on the Bernoulli convolutions related to $\beta$-numeration for $1<\beta<\mathbf{d}$, where $\mathbf{d} \geqslant 2$ is an integer. The set $\mathscr{D}^{\mathbf{N}}$ of sequences $\omega=\left(\omega_{k}\right)_{k=0}^{\infty}$ with digits $\omega_{k}$ in the finite alphabet $\mathscr{D}:=\{\mathbf{0}, \ldots, \mathbf{d}-\mathbf{1}\}$ is endowed with the product topology. Given a d-dimensional probability vector $\mathrm{p}:=\left(\mathrm{p}_{i}\right)_{i=0}^{\mathbf{d}-\mathbf{1}}$, the $\mathrm{p}$-distributed $(\beta, \mathbf{d})$-Bernoulli convolution is by definition the measure $\mu$ which corresponds to the distribution of the random variable $X: \mathscr{D}^{\mathbf{N}} \rightarrow \mathbf{R}$ such that $X(\omega)=\sum_{k=0}^{\infty} \omega_{k} / \beta^{k+1}$, where $\omega \mapsto \omega_{k}(k=0,1, \ldots)$ is a sequence of i.i.d. random

\footnotetext{
* This paper is dedicated to the memory of Jean Marie Dumont.

$\dagger$ Supported by a HK RGC grant and a CUHK Postdoctoral Fellowship.

$\ddagger$ Supported by the EPSRC grant no GR/R61451/01.
} 
variables, assuming the value $i \in\{\mathbf{0}, \mathbf{1}, \ldots, \mathbf{d}-\mathbf{1}\}$ with the probability $\mathrm{p}_{i}$. We speak of the uniform Bernoulli convolution when $\mathrm{p}_{i}=1 / \mathbf{d}$ for each $i$.

The measure $\mu$ is self-similar and, thus, satisfies the so-called law of pure types. Recall that this means that $\mu$ is either absolutely continuous or purely singular with respect to the Lebesgue measure. Moreover, when each $\mathrm{p}_{i}$ is positive, the measure $\mu$ is fully supported by an interval.

Because of their nontrivial multifractal structure, we will consider the Bernoulli convolutions known to be purely singular, namely, those parameterized by Pisot-Vijayaraghavan (PV) number $\beta$. In Section 2 we use an important arithmetic property of PV numbers (Garsia's separation lemma) to obtain, in Lemma 2.2, a decomposition of the Bernoulli measure $\mu$ which involves a matrix product (see [16] for a similar approach). Moreover, from the $\beta$-shift being of finite type it follows (Sections 2.3 and 2.4) that $\mu$ may be decomposed into a finite number of measures having a specific structure; we call them $\mathscr{M}$-measures, where $\mathscr{M}$ is a finite set of square matrices with nonnegative entries.

In Section 3 we consider the case when $\mu$ is the $(\beta, 2)$-Bernoulli convolution, where $\beta$ is a multinacci number of order $\mathbf{m} \geqslant 2$ (see the definition below). The main result of this section (Theorem 3.2) concerns the "local" Gibbs properties of $\mu$.

Finally, in Section 4 we show that the "local" Gibbs properties allow one to apply the multifractal analysis of $\mu$ (see Theorem 4.2). Moreover, in the same section we show how to use the multifractal analysis as a classification tool and discuss the existence of "global" Gibbs properties of $\mu$ in the cases of the uniform/nonuniform Erdös measure $(\mathbf{m}=2$ and $\beta=(1+\sqrt{5}) / 2)$ - Theorems $4.5,4.7$ and 4.8 .

\section{Basic Notions: Definitions and Generalities}

1.1. Net and adapted system of affine contractions. For a given integer $s \geqslant 2$ we consider the finite alphabet $\mathscr{A}:=\{\mathbf{0}, \ldots, \mathbf{s}-\mathbf{1}\}$ and call any element $w=$ $\xi_{0} \cdots \xi_{n-1} \in \mathscr{A}^{n}(n \geqslant 1)$ a word. By convention, $\mathscr{A}^{0}:=\{\phi\}$, where $\phi$ denotes the empty word. Let $\mathscr{A}^{*}$ denote the set of all words in the alphabet $\mathscr{A}$, i.e., $\mathscr{A}^{*}:=$ $\bigcup_{n=0}^{\infty} \mathscr{A}^{n}$. The concatenation of the two words $w, w^{\prime}$ is, as usual, denoted by $w w^{\prime}$.

The family $\mathfrak{F}:=\left\{\mathfrak{F}_{n}\right\}_{n=0}^{\infty}$ is called an s-fold net of the interval $[a, b]$ if $\mathfrak{F}_{n}$ is a partition of $\left[a, b\left[\right.\right.$ by $\mathbf{s}^{n}$ semi-open intervals (which we denote by $\llbracket w \rrbracket$ for $w \in \mathscr{A}^{n}$ ) with the extra property that $\llbracket w w^{\prime} \rrbracket \subset \llbracket w \rrbracket$, for any words $w, w \in \mathscr{A}^{*}$. Any interval $\llbracket w \rrbracket$ is by definition a basic interval of the net $\mathfrak{F}$. By Kolmogorov's Consistency Theorem, any positive Borel measure $\eta$ on the real line whose support is a subset of $[a, b]$, is characterized by its values taken on the intervals of the s-fold net.

Let $\mathscr{S}:=\left\{S_{i}\right\}_{i=0}^{\mathbf{s}-\mathbf{1}}$ be a system of (orientation preserving) affine contractions (s.a.c.) of the real line; we say that $\mathscr{S}$ is adapted to the interval $[a, b[$ when $\left\{\mathbb{S}_{i}\left[a, b[\}_{i=0}^{\mathbf{s}-\mathbf{1}}\right.\right.$ is a partition of $\left[a, b\left[\right.\right.$. By convention, $S_{\phi}$ is the identity map on $\mathbf{R}$ and, for any non-empty word $w=\xi_{0} \cdots \xi_{n-1} \in \mathscr{A}^{*}$, we put $S_{w}:=S_{\xi_{0}} \circ \cdots \circ S_{\xi_{n-1}}$. The $\mathscr{S}$-net is by definition the s-fold net $\mathfrak{F}:=\left\{\mathfrak{F}_{n}\right\}_{n=0}^{\infty}$, where $\mathfrak{F}_{n}$ is the collection of the intervals $\llbracket w \rrbracket:=\mathbb{S}_{w}\left[a, b\left[\right.\right.$ for $w \in \mathscr{A}^{n}$.

1.2. $\mathscr{M}$-measures. Given $\mathscr{M}:=\left\{M_{i}\right\}_{i=0}^{\mathrm{s}-\mathbf{1}}$, a family of $r \times r$ matrices $(r \geqslant 1)$ with nonnegative entries, we denote $M_{*}=M_{0}+\cdots+M_{\mathbf{s}-\mathbf{1}}$. Assume there exists a 
column-vector $R \neq 0$ with nonnegative entries such that $M_{*} R=R$; we say that $\nu$ is a $\mathscr{M}$-measure w.r.t. the s-fold net $\mathfrak{F}$ if its support is a subset of $[a, b]$ and if there exists a row-vector $L$ with nonnegative entries such that $\nu \llbracket w \rrbracket=L M_{w} R$, for any word $w=$ $\xi_{0} \cdots \xi_{n-1} \in \mathscr{A}^{*}$ (by a similar convention as above, $M_{\phi}$ is the unit $r \times r$ matrix and $\left.M_{w}:=M_{\xi_{0}} \cdots M_{\xi_{n-1}}\right)$.

1.3. Gibbs, weak Gibbs and quasi-Bernoulli measures. Let $\eta$ be a finite positive Borel measure whose support is $[a, b]$. We denote by $\sigma: \mathscr{A}^{\mathbf{N}} \rightarrow \mathscr{A}^{\mathbf{N}}$ the one-sided shift map defined for any $\xi=\left(\xi_{k}\right)_{k=0}^{\infty} \in \mathscr{A}^{\mathbf{N}}$ by $\sigma \xi=\left(\xi_{k+1}\right)_{k=0}^{\infty}$. Recall that the product topology on $\mathscr{A}^{\mathbf{N}}$ is given by the metric such that the distance between $\xi$ and $\zeta$ is $2^{-k}$, where $k$ is the length of the largest common prefix of $\xi$ and $\zeta$.

Definition 1.1. The measure $\eta$ is $\mathfrak{F}$-weak Gibbs in the sense of Yuri [32] if there exists a continuous map $\Phi$ from $\mathscr{A}^{\mathbf{N}}$ to $\mathbf{R}$ (called a potential) and a sequence of real numbers $K_{n}>1$, subexponential in the sense that $\lim _{n} \frac{1}{n} \log K_{n}=0$ such that, for any $\xi \in \mathscr{A}^{\mathbf{N}}$ and any $n \geqslant 1$,

$$
\frac{1}{K_{n}} \leqslant \frac{\eta \llbracket \xi_{0} \cdots \xi_{n-1} \rrbracket}{\exp \left(\sum_{k=0}^{n-1} \Phi\left(\sigma^{k} \xi\right)\right)} \leqslant K_{n} .
$$

When a sequence $\left(K_{n}\right)$ can be taken constant, $\eta$ is $\mathfrak{F}$-Gibbs in the sense of Bowen [3].

For each $\xi \in \mathscr{A}^{\mathbf{N}}$ we put $\phi_{1}(\xi)=\log \eta \llbracket \xi_{0} \rrbracket$ and for $n \geqslant 2$,

$$
\phi_{n}(\xi)=\log \left(\frac{\eta \llbracket \xi_{0} \cdots \xi_{n-1} \rrbracket}{\eta \llbracket \xi_{1} \cdots \xi_{n-1} \rrbracket}\right) .
$$

The continuous map $\phi_{n}: \mathscr{A}^{\mathbf{N}} \mapsto \mathbf{R}(n \geqslant 1)$ is called the $n$-step potential of $\eta$. Assume that the sequence $\phi_{n}$ converges uniformly to a potential $\Phi$; it is then straightforward that for $n \geqslant 1$,

$$
\frac{1}{K_{n}} \leqslant \frac{\eta \llbracket \xi_{0} \cdots \xi_{n-1} \rrbracket}{\exp \left(\sum_{k=0}^{n-1} \Phi\left(\sigma^{k} \xi\right)\right)} \leqslant K_{n} \quad \text { with } K_{n}=\exp \left(\sum_{k=1}^{n}\left\|\Phi-\phi_{n}\right\|_{\infty}\right) .
$$

By a well known lemma on the Cesàro sums, the sequence $\left(K_{n}\right)_{n}$ is subexponential, whence (3) means $\eta$ is $\mathfrak{F}$-weak Gibbs. Note that if $\sum_{n}\left\|\Phi-\phi_{n}\right\|_{\infty}<+\infty$, then the $K_{n}$ are bounded and therefore, $\eta$ is a $\mathfrak{F}$-Gibbs measure.

We also consider quasi-Bernoulli measures.

Definition 1.2. The positive measure $\eta$ whose support is a subset of the interval $[a, b]$, is said to be $\mathfrak{F}$-quasi-Bernoulli if there exists a constant $K>1$ such that for any words $w, w^{\prime} \in \mathscr{A}^{*}$,

$$
\frac{1}{K} \eta \llbracket w \rrbracket \eta \llbracket w^{\prime} \rrbracket \leqslant \eta \llbracket w w^{\prime} \rrbracket \leqslant K \eta \llbracket w \rrbracket \eta \llbracket w^{\prime} \rrbracket .
$$

The net $\mathfrak{F}$ itself is said quasi-Bernoulli if the Lebesgue measure (restricted to $[a, b])$ is $\mathscr{\mho}$-quasi-Bernoulli.

Notice that a Gibbs measure is always quasi-Bernoulli. 
1.4. Multifractal analysis. We need a number of extra definitions. Recall that the local dimension at a point $x$ which belongs to the support of the measure $\eta$, is $\lim _{r \rightarrow 0} \log \eta\left(B_{r}(x)\right) / \log r$, provided the limit exists. (Here $B_{r}(x)$ stands for the closed ball of radius $r$ centered at $x$.) Given an arbitrary real $\alpha$, the level set $E_{\eta}(\alpha)$ is defined as the set of $x$ in the support of $\eta$ such that the local dimension at $x$ exists and is equal to $\alpha$. The multifractal domain $\operatorname{Dom}(\eta)$ is the set of $\alpha \in \mathbf{R}$ for which $E_{\eta}(\alpha)$ is nonempty. The singularity spectrum is the map which associates to any $\alpha \in \mathbf{R}$ the Hausdorff dimension $\operatorname{dim}_{H} E_{\eta}(\alpha)$. The scale spectrum (also called $L^{q}$-spectrum) is the map $\tau_{\eta}$ from $\mathbf{R}$ to $\mathbf{R} \cup\{+\infty\}$ defined as follows:

$$
\tau_{\eta}(q):=\liminf _{r \rightarrow 0} \frac{\log \inf \left\{\sum_{i} \eta\left(J_{i}\right)^{q} ;\left\{J_{i}\right\}_{i}\right\}}{\log r},
$$

where $\left\{J_{i}\right\}_{i}$ runs over the family of covers of the support of $\eta$ by closed intervals $J_{i}$ whose length is equal to $r$. (We refer to the Book of Pesin [25] for analogue and equivalent definitions of the scale spectrum.)

One usually says that $\eta$ satisfies the multifractal formalism if the singularity spectrum and the scale spectrum of $\eta$ form a Legendre transform pair. The multifractal formalism is trivially not universal, but it has been established for wide classes of measures [28, 4, 10, 26, 22], when some conditions of geometric homogeneity are satisfied (self-similarity, conformality or Gibbs properties, etc.). For our purpose, we refer to the multifractal formalism of the quasi-Bernoulli and weak Gibbs measures stated in the two following theorems:

Theorem 1.3 [4, 9]. Let $\mathfrak{E}$ be an s-fold quasi-Bernoulli net of a compact interval $[a, b]$ and $\eta$ be a positive measure fully supported by $[a, b]$. If $\eta$ is a E-quasi-Bernoulli measure, then

1. The scale spectrum $\tau_{\eta}$ of $\eta$ is concave and differentiable on the whole real line and moreover,

$$
-\infty<\underline{\alpha}:=\lim _{q \rightarrow+\infty} \frac{\tau_{\eta}(q)}{q} \leqslant \lim _{q \rightarrow-\infty} \frac{\tau_{\eta}(q)}{q}=: \bar{\alpha}<+\infty .
$$

2. The multifractal domain of $\eta$ is $\operatorname{Dom}(\eta)=[\underline{\alpha} ; \bar{\alpha}]$ and for any $\underline{\alpha} \leqslant \alpha \leqslant \bar{\alpha}$,

$$
\operatorname{dim}_{H} E_{\eta}(\alpha)=\inf _{q \in \mathbf{R}}\left\{\alpha q-\tau_{\eta}(q)\right\}
$$

meaning that $\eta$ satisfies the multifractal formalism.

Theorem 1.4 [7, Theorem $\left.\mathrm{A}^{\prime}\right]$. Let $\mathfrak{E}$ be an $\mathbf{s}$-fold net of a compact interval $[a, b]$ with respect to which the Lebesgue measure is Gibbs. If the positive measure $\eta$, supposed to be fully supported by $[a, b]$, is weak Gibbs w.r.t. $\mathfrak{E}$, then

1. The scale spectrum $\tau_{\eta}$ of $\eta$ is concave on the whole real line and moreover,

$$
-\infty<\underline{\alpha}:=\lim _{q \rightarrow+\infty} \frac{\tau_{\eta}(q)}{q} \leqslant \lim _{q \rightarrow-\infty} \frac{\tau_{\eta}(q)}{q}=: \bar{\alpha}<+\infty .
$$

2. The multifractal domain of $\eta$ is $\operatorname{DOM}(\eta)=[\underline{\alpha} ; \bar{\alpha}]$ and for any $\underline{\alpha} \leqslant \alpha \leqslant \bar{\alpha}$,

$$
\operatorname{dim}_{H} E_{\eta}(\alpha)=\inf _{q \in \mathbf{R}}\left\{\alpha q-\tau_{\eta}(q)\right\}
$$

meaning that $\eta$ satisfies the multifractal formalism. 
Remark 1.5. The analysis of the Gibbs properties of a given measure is a simple way to study its multifractal structure. Conversely, the multifractal properties of the measure may be used as a classification tool w.r.t. its Gibbs properties.

For instance, a Gibbs measure is both weak Gibbs and quasi-Bernoulli, but a weak Gibbs measure need not be quasi-Bernoulli, for there exist weak Gibbs measures, whose scale spectrum is not differentiable (see $[6,7]$ ).

Another interesting application uses topological properties of the multifractal domain for the measure in question. Actually, a measure whose multifractal domain is disconnected (or noncompact) is neither weak Gibbs nor quasiBernoulli w.r.t. any given reasonable net.

Below we discuss these types of classification for the cases of the uniform/ nonuniform Erdős measure, see Theorems 4.7 and 4.8.

\section{Decomposition of Bernoulli Convolutions}

2.1. Generalities. Let $\mathbf{b}, \mathbf{d}$ be two integers and $\beta$ a real number such that

$$
1 \leqslant \mathbf{b}-1<\beta \leqslant \mathbf{b} \leqslant \mathbf{d} .
$$

Throughout this paper, we consider the following two alphabets:

$$
\mathscr{B}:=\{\mathbf{0}, \ldots, \mathbf{b}-1\} \quad \text { and } \mathscr{D}:=\{\mathbf{0}, \ldots, \mathbf{d}-1\} .
$$

Recall that the $\mathbf{p}$-distributed $(\beta, \mathbf{d})$-Bernoulli convolution is the measure $\mu$ such that, for any Borel set $B \subset \mathbf{R}$,

$$
\mu(B)=\mathbb{P}\{\omega: X(\omega) \in B\},
$$

where $X(\omega)=\sum_{k=0}^{\infty} \omega_{k} / \beta^{k+1}$ for any $\omega \in \mathscr{D}^{\mathbf{N}}$, and $\mathbb{P}$ is the product measure on $\mathscr{D}^{\mathbf{N}}$ with parameter $p=\left(p_{0}, \ldots, p_{d-1}\right)$.

The $\beta$-numeration is usually related to the s.a.c. $\left\{\mathbb{R}_{i}\right\}_{i=0}^{\mathbf{b}-1}$ defined as follows:

$$
\mathbb{R}_{i}(x)=(x+i) / \beta \text {. }
$$

From now on, $i$ will always stand for an arbitrary fixed element in $\mathscr{B}$; denoting by $\sigma: \mathscr{D}^{\mathbf{N}} \rightarrow \mathscr{D}^{\mathbf{N}}$ the shift map on the product space $\mathscr{D}^{\mathbf{N}}$, for any Borel set $B \subset \mathbf{R}$, and $x \in \mathbf{R}$, one has $X(\omega) \in B+x$ if and only if $X(\sigma \omega) \in \mathbb{R}_{i}^{-1}(B)+\beta x+\left(i-\omega_{0}\right)$. Since $\mathbb{P}$ is a product measure,

$$
\mu(B+x)=\sum_{j=\mathbf{0}}^{\mathbf{d}-1} \mathrm{p}_{j} \cdot \mu\left(\mathbb{R}_{i}^{-1}(B)+\beta x+(i-j)\right) .
$$

Suppose that $\mathbb{R}_{i}^{-1}(B) \subset[0,1]$; the support of $\mu$ being a subset of $\left[0, \alpha_{\mu}\right]$ with $\alpha_{\mu}:=(\mathbf{d}-1) /(\beta-1)$, one has $\mu\left(\mathbb{R}_{i}^{-1}(B)+y\right)=0$ whenever $\left.y \notin\right]-1, \alpha_{\mu}[$. Given any $x \in]-1, \alpha_{\mu}[$, we write,

$$
\stackrel{i}{\triangleright \triangleright y} \Longleftrightarrow-1<y<\alpha_{\mu} \quad \text { and } \quad \beta x+i-y \in \mathscr{D}
$$

and

$$
x \triangleright y \Longleftrightarrow \exists i \in \mathscr{B}, \quad \stackrel{i}{\triangleright} \triangleright y .
$$

Then, in order to simplify the relation (4), we set the following definition: 
Definition 2.1. The real number $r$ belongs to the set $\mathscr{I}_{(\beta, \mathbf{d})}$ if there exist a finite sequence of real numbers $0=r_{0}, \ldots, r_{n}=r$ such that $r_{0} \triangleright r_{1} \triangleright \cdots \triangleright r_{n}$.

The set $\mathscr{I}_{(\beta, \mathbf{d})}$ is always nonempty, because it contains at least 0 ; moreover, it is clearly finite or countable and we denote by $0=\mathrm{i}_{0}, \mathrm{i}_{1}, \ldots$ the sequence of its elements. Actually, in order to determine $\mathscr{I}_{(\beta, \mathbf{d})}$ explicitly, we present an equivalent definition using induction (see also [17] and [2, Section 2]).

Firstly, since $0=\mathrm{i}_{0} \in \mathscr{I}_{(\beta, \mathbf{d})}$, we define $\mathscr{I}_{0}:=\left\{\mathrm{i}_{0}\right\}$; then, assuming that $\mathscr{I}_{n}=\left\{\mathrm{i}_{0}, \ldots, \mathrm{i}_{k_{n}}\right\}$, we put

$$
\mathscr{I}_{n+1}:=\bigcup_{k=0}^{k_{n}}\left\{y=\beta \mathbf{i}_{k}+(i-j) ;(i, j) \in \mathscr{B} \times \mathscr{D} \text { and }-1<y<\alpha_{\mu}\right\},
$$

and finally,

$$
\mathscr{I}_{(\beta, \mathbf{d})}:=\bigcup_{n} \mathscr{I}_{n} .
$$

Notice that $\mathscr{I}_{(\beta, \mathbf{d})}$ is finite whenever $\mathscr{I}_{n-1}=\mathscr{I}_{n}$ for some $n \geqslant 1$; in that case, $\mathscr{I}_{(\beta, \mathbf{d})}=\mathscr{I}_{n}$.

It follows from (4) that, for $B \subset[0,1]$ with $\mathbb{R}_{i}^{-1}(B) \subset[0,1]$,

$$
\mu\left(B+\mathrm{i}_{h}\right)=\sum_{k} M_{i}(h, k) \mu\left(\mathbb{R}_{i}^{-1}(B)+\mathrm{i}_{k}\right)
$$

where $M_{i}$ is the nonnegative matrix defined as follows: for the row index $h$ and the column index $k$, with $\dot{i}_{h}$ and $\dot{i}_{k}$ in $\mathscr{I}_{(\beta, \mathbf{d})}$, by definition,

$$
M_{i}(h, k)= \begin{cases}\mathrm{p}_{j}, & \text { if } j=i+\beta \mathrm{i}_{h}-\mathrm{i}_{k} \in \mathscr{D} \\ 0, & \text { otherwise. }\end{cases}
$$

Lemma 2.2. Let $i \in \mathscr{B}$ and assume $\mathscr{I}_{(\beta, \mathbf{d})}:=\left\{\mathrm{i}_{0}, \ldots, \mathrm{i}_{\mathbf{r}-1}\right\}$; then, for any Borel set $B \subset[0,1]$ with $\mathbb{R}_{i}^{-1}(B) \subset[0,1]$, one has:

$$
\left(\begin{array}{c}
\mu\left(B+\mathrm{i}_{0}\right) \\
\vdots \\
\mu\left(B+\mathrm{i}_{\mathbf{r}-1}\right)
\end{array}\right)=M_{i}\left(\begin{array}{c}
\mu\left(\mathbb{R}_{i}^{-1}(B)+\mathrm{i}_{0}\right) \\
\vdots \\
\mu\left(\mathbb{R}_{i}^{-1}(B)+\mathrm{i}_{\mathbf{r}-1}\right)
\end{array}\right) .
$$

Example 2.3. Let $\beta$ be the algebraic integer such that $\beta^{2}=5 \beta+3$ with $5<\beta<6$ and $\mathbf{d}=6$, so that $\mathscr{B}=\mathscr{D}=\{\mathbf{0}, \ldots, \mathbf{5}\}$. We apply the argument described above to determine the set $\mathscr{I}_{(\beta, 6)}$ and the matrices $M_{\mathbf{0}}, \ldots, M_{\mathbf{5}}$. Following the induction process leading to the definition in (5), one has $\mathscr{I}_{0}=\left\{\mathbf{i}_{0}=0\right\}$ and using the fact that $1<\alpha_{\mu}=5 /(\beta-1)<2$, one obtains successively:

$$
\begin{aligned}
\mathscr{I}_{1} & =\mathscr{I}_{0} \cup\left\{y=i-j ;(i, j) \in \mathscr{B} \times \mathscr{D} \text { and }-1<y<\alpha_{\mu}\right\} \\
& =\left\{\mathrm{i}_{0}=0, \mathrm{i}_{1}=1\right\} \quad(\text { with } 0 \triangleright 0 \text { and } 0 \triangleright 1) \\
\mathscr{I}_{2} & =\mathscr{I}_{1} \cup\left\{y=\beta+(i-j) ;(i, j) \in \mathscr{B} \times \mathscr{D} \text { and }-1<y<\alpha_{\mu}\right\} \\
& =\left\{\mathrm{i}_{0}=0, \mathrm{i}_{1}=1, \mathrm{i}_{2}=\beta-5\right\}(\text { with } 1 \triangleright \beta-5) \\
\mathscr{I}_{3} & =\mathscr{I}_{2} \cup\left\{y=\beta(\beta-5)+(i-j) ;(i, j) \in \mathscr{B} \times \mathscr{D} \text { and }-1<y<\alpha_{\mu}\right\} \\
& =\mathscr{I}_{2}=\mathscr{I}_{(\beta, 6)}(\text { with } \beta-5 \triangleright 0 \text { and } \beta-5 \triangleright 1)
\end{aligned}
$$




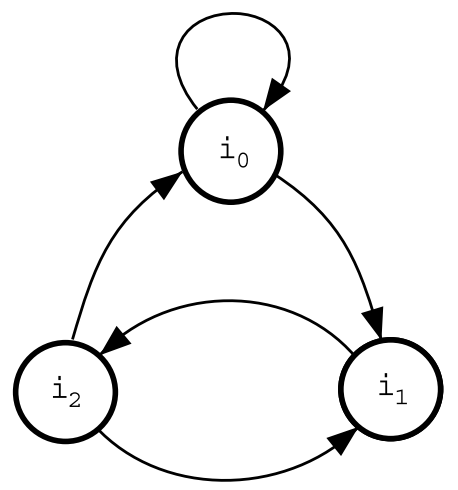

Figure 1. In the case of the PV number $\beta$ such that $\beta^{2}=5 \beta+3$, the set $\mathscr{I}_{(\beta, 6)}$ has three elements, say $\mathrm{i}_{0}=0, \mathrm{i}_{1}=1$ and $\mathrm{i}_{2}=\beta-5$; here, we give a representation of the graph of the relation $\cdot \triangleright \cdot$ on $\mathscr{I}_{(\beta, 6)}$

Finally, $\mathscr{I}_{(\beta, 6)}=\left\{\mathrm{i}_{0}=0, \mathrm{i}_{1}=1, \mathrm{i}_{2}=\beta-5\right\}$. Figure 1 shows the graph of the relation $\cdot \triangleright \cdot$ on $\mathscr{I}_{(\beta, 6)}$. Moreover, the algorithm we use to determine the set $\mathscr{I}_{(\beta, 6)}$ provides us with extra information sufficient to obtain the matrices $M_{\mathbf{0}}, \ldots, M_{\mathbf{5}}$ as defined in (7) - see Fig. 2 . We thus have (for $i=\mathbf{0}, \ldots, \mathbf{5}$ ):

$$
M_{i}=\left(\begin{array}{ccc}
\mathrm{p}_{i} & \mathrm{p}_{i-1} & 0 \\
0 & 0 & \mathrm{p}_{i+5} \\
\mathrm{p}_{i+3} & \mathrm{p}_{i+2} & 0
\end{array}\right),
$$

where, by convention, $\mathrm{p}_{i}=0$, for any $i \leqslant-1$ or $i \geqslant 6$ (see [20] for the general case when $\beta$ is a quadratic number).

Example 2.4. Assume now $\beta$ to be the PV number satisfying $\beta^{3}=3 \beta^{2}-1$. The set $\mathscr{I}_{(\beta, 3)}$ has then eight elements which we present in the form of the list:

$$
\begin{array}{llll}
\mathbf{i}_{0}=0, & \mathbf{i}_{1}=1, & \mathbf{i}_{2}=\beta-2, & \mathbf{i}_{3}=\beta^{2}-2 \beta-2, \\
\mathbf{i}_{4}=\beta^{2}-2 \beta-3, & \mathbf{i}_{5}=\beta^{2}-3 \beta, & \mathbf{i}_{6}=\beta^{2}-3 \beta+1, & \mathbf{i}_{7}=\beta-3 .
\end{array}
$$

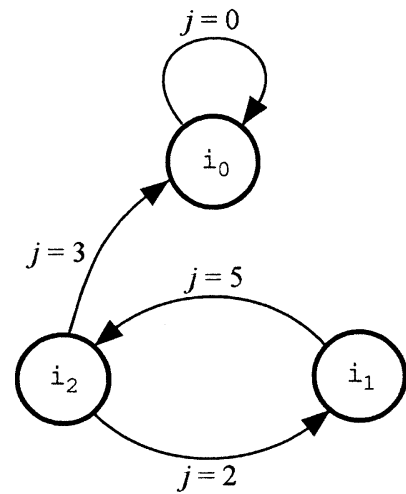

Automaton of $\cdot \stackrel{i}{\triangleright} \cdot$ with $i=0$

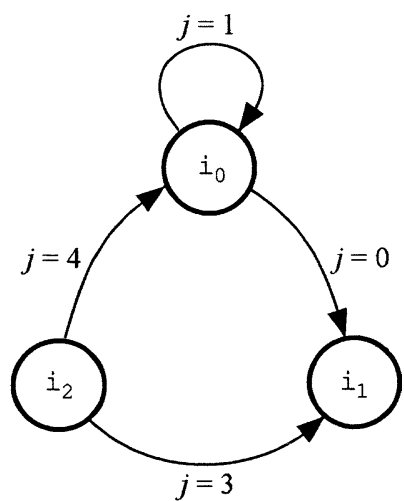

Automaton of $\stackrel{i}{\triangleright}$. with $i=\mathbf{1}$

Figure 2. We represent here, the automaton of the relation $\cdot \stackrel{i}{\triangleright} \cdot$ for $i=\mathbf{0}$ and $\mathbf{1}$ (the cases $i=\mathbf{2}, \ldots, \mathbf{5}$ may be recovered by mean of the matrices in (8)). When $\mathrm{i}_{h} \stackrel{i}{\triangleright} \mathrm{i}_{k}$, the label $j$ of the arrow from state $\mathrm{i}_{h}$ to state $\mathbf{i}_{k}$, means that $j=\beta \mathbf{i}_{h}-\mathbf{i}_{k}+i$; then, $j \in \mathscr{D}$ and according to (7) one has $M_{i}(h, k)=\mathrm{p}_{j}$ 


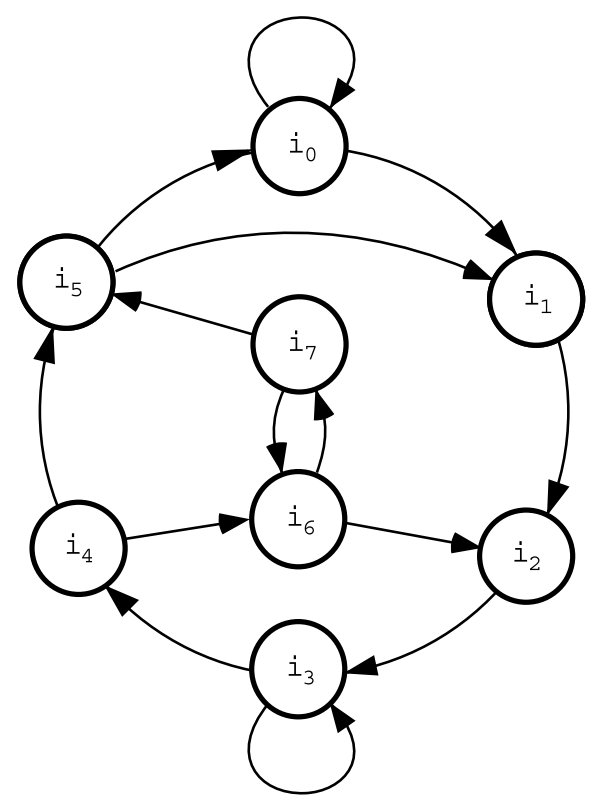

Figure 3. In the case of the PV number $\beta$ such that $\beta^{3}=3 \beta^{2}-1$, the set $\mathscr{I}_{(\beta, 3)}$ has eight elements listed in (9); here, we give a representation of the graph of the relation $\cdot \triangleright \cdot$ on $\mathscr{I}_{(\beta, 3)}$

The graph of the relation $\cdot \triangleright \cdot$ on $\mathscr{I}_{(\beta, 3)}$, is represented in Fig. 3 .

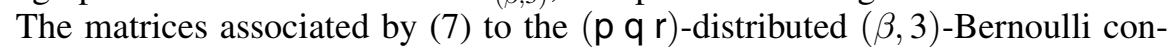
volution are:

$$
\begin{gathered}
M_{\mathbf{0}}=\left(\begin{array}{cccccccc}
\mathrm{p} & 0 & 0 & 0 & 0 & 0 & 0 & 0 \\
0 & 0 & \mathrm{r} & 0 & 0 & 0 & 0 & 0 \\
0 & 0 & 0 & \mathrm{r} & 0 & 0 & 0 & 0 \\
0 & 0 & 0 & \mathrm{q} & \mathrm{r} & 0 & 0 & 0 \\
0 & 0 & 0 & 0 & 0 & 0 & 0 & 0 \\
0 & 0 & 0 & 0 & 0 & 0 & 0 & 0 \\
0 & 0 & \mathrm{q} & 0 & 0 & 0 & 0 & \mathrm{r} \\
0 & 0 & 0 & 0 & 0 & \mathrm{p} & 0 & 0
\end{array}\right) \quad M_{\mathbf{1}}=\left(\begin{array}{cccccccccc}
\mathrm{q} & \mathrm{p} & 0 & 0 & 0 & 0 & 0 & 0 \\
0 & 0 & 0 & 0 & 0 & 0 & 0 & 0 \\
0 & 0 & 0 & 0 & 0 & 0 & 0 & 0 \\
0 & 0 & 0 & \mathrm{r} & 0 & 0 & 0 & 0 \\
0 & 0 & 0 & 0 & 0 & \mathrm{p} & 0 & 0 \\
\mathrm{p} & 0 & 0 & 0 & 0 & 0 & 0 & 0 \\
0 & 0 & \mathrm{r} & 0 & 0 & 0 & 0 & 0 \\
0 & 0 & 0 & 0 & 0 & \mathrm{q} & \mathrm{p} & 0
\end{array}\right) \\
M_{2}=\left(\begin{array}{cccccccc}
\mathrm{r} & \mathrm{q} & 0 & 0 & 0 & 0 & 0 & 0 \\
0 & 0 & 0 & 0 & 0 & 0 & 0 & 0 \\
0 & 0 & 0 & 0 & 0 & 0 & 0 & 0 \\
0 & 0 & 0 & 0 & 0 & 0 & 0 & 0 \\
0 & 0 & 0 & 0 & 0 & \mathrm{q} & \mathrm{p} & 0 \\
\mathrm{q} & \mathrm{p} & 0 & 0 & 0 & 0 & 0 & 0 \\
0 & 0 & 0 & 0 & 0 & 0 & 0 & 0 \\
0 & 0 & 0 & 0 & 0 & \mathrm{r} & \mathrm{q} & 0
\end{array}\right) . \\
\end{gathered}
$$


2.2. Known cases when $\mathscr{I}_{(\beta, \mathbf{d})}$ is finite. Our analysis of a $(\beta, \mathbf{d})$-Bernoulli convolution relies on the finiteness of $\mathscr{I}_{(\beta, \mathbf{d})}$; for instance, this is trivially the case when $\beta$ is an integer:

Proposition 2.5. If $\beta$ is an integer one has $\mathscr{I}_{(\beta, \mathbf{d})}=\{\mathbf{0}, \ldots, \mathbf{a}-1\}$, where $\mathbf{a}$ is the integer such that $\mathbf{a}-1<(\mathbf{d}-1) /(\beta-1) \leqslant \mathbf{a}$.

This proposition is the starting point of our analysis of the Bernoulli convolution in an integral basis developed in [21]; however, the methods used in that paper are different from the ones we use here.

The second case for which we know that $\mathscr{I}_{(\beta, \mathbf{d})}$ is finite arises when $\beta$ is a noninteger PV number, i.e., an algebraic integer $\beta=\beta_{s}>1$ whose Galois conjugates $\beta_{1}, \ldots, \beta_{s-1}$ are strictly less than 1 in modulus. Given any polynomial $A(X) \in \mathbf{Z}[X]$ such that $A(\beta) \neq 0$, it is necessary that $A\left(\beta_{k}\right) \neq 0$ for $k=1, \ldots, s$ and thus the integer $\left|A\left(\beta_{1}\right) \cdots A\left(\beta_{s}\right)\right|$ is greater or equal to 1 . Since $\left|\beta_{k}\right|<1$ for $k=1, \ldots, s-1$, one deduces that $\left|A\left(\beta_{k}\right)\right| \leqslant M /\left(1-\left|\beta_{k}\right|\right)$, where $M$ is the maximum of the absolute values of the coefficients of $A(X)$ : in other words,

$$
\forall A(X) \in \mathbf{Z}[X], \quad A(\beta) \neq 0 \Rightarrow|A(\beta)| \geqslant \frac{1}{M^{s-1}} \prod_{k=1}^{s-1}\left(1-\left|\beta_{k}\right|\right)>0 .
$$

The fact that (10) is satisfied by the PV numbers has been discovered by Garsia [8] and is usually called Garsia's separation lemma (see also [16]).

Return to the question of the cardinality of $\mathscr{I}_{(\beta, \mathbf{d})}$ and note that for any element $i$ in this set there exists a finite sequence $\varepsilon_{0}, \ldots, \varepsilon_{m}$ of integers lying between $-\mathbf{d}$ and $\mathbf{b}$ such that $i=\varepsilon_{0}+\varepsilon_{1} \beta+\cdots+\varepsilon_{m} \beta^{m}$. Since $\beta$ is a PV number, it follows from (10) that the distance between two different elements in $\mathscr{I}_{(\beta, \mathbf{d})}$ is bounded from below by $(2 \mathbf{d})^{1-s} \prod_{k=1}^{s-1}\left(1-\left|\beta_{k}\right|\right)$, which yields the following proposition:

Proposition 2.6. The set $\mathscr{I}_{(\beta, \mathbf{d})}$ is finite when $\beta$ is a PV number.

Proposition 2.7. If $\mathscr{I}_{(\beta, \mathbf{d})}$ is finite, then all the conjugates of $\beta$ are less than $\beta$ in modulus and also less in modulus than $(1+\sqrt{5}) / 2$.

The proof immediately follows from the fact that $\mathscr{I}_{(\beta, \mathbf{d})}$ contains the orbit of 1 under the $\beta$-shift; then we may use the results of Solomyak [31]. We leave details to the reader.

Remark 2.8. It should be interesting to find a non-PV number $\beta$ for which $\mathscr{I}_{(\beta, \mathbf{d})}$ is finite, even when (10) does not hold. We believe the only possible counterexample may be a Salem number (the one for which all its conjugates are less than or equal to 1 in modulus and some of them are equal to 1 in modulus).

2.3. Heuristics. In this section we give a heuristic description of the framework that we use in Section 3 for the complete analysis of the Bernoulli convolution in the multinacci bases.

Recall that a $(\beta, \mathbf{d})$-Bernoulli convolution $\mu$ is supported by the interval $\left[0, \alpha_{\mu}\right]$ with $\alpha_{\mu}=(\mathbf{d}-1) /(\beta-1)$. We assume that $\mathscr{I}_{(\beta, \mathbf{d})}=\left\{\mathrm{i}_{0}, \ldots, \mathrm{i}_{\mathrm{r}-1}\right\}$ and for any 
$j=0, \ldots, \mathbf{r}-1$, we define the measure $\mu_{j}$ by putting, for any Borel set $B$ of the real line,

$$
\mu_{j}(B)=\mu\left(B \cap[0,1]+\mathbf{i}_{j}\right) .
$$

If both $B$ and $\mathbb{R}_{i}^{-1}(B)(i \in \mathscr{B})$ are subsets of $[0,1]$, it follows from Lemma 2.2 that

$$
\left(\begin{array}{c}
\mu_{0}(B) \\
\vdots \\
\mu_{\mathbf{r}-1}(B)
\end{array}\right)=M_{i}\left(\begin{array}{c}
\mu_{0}\left(\mathbb{R}_{i}^{-1}(B)\right) \\
\vdots \\
\mu_{\mathbf{r}-1}\left(\mathbb{R}_{i}^{-1}(B)\right)
\end{array}\right)
$$

Our second assumption is the existence of $\mathbf{s}$ words $w_{\mathbf{0}}, \ldots, w_{\mathbf{s}-\mathbf{1}}$ in $\mathscr{B}^{*}$ which satisfy the two conditions: firstly, $\mathbb{R}_{w}[0,1] \subset[0,1]$, for any suffix $w$ of any of the words $w_{j}$, for $j=\mathbf{0}, \ldots, \mathbf{s}-\mathbf{1}$; secondly, the s.a.c. $\mathscr{R}:=\left\{\hat{\mathbb{R}}_{j}=\mathbb{R}_{w_{j}}\right\}_{j=\mathbf{0}}^{s-1}$ is adapted to the interval $[0,1]$.

The s.a.c. $\mathscr{R}$ is associated to an s-fold net of $[0,1[$, say $\mathscr{F}$, whose basic intervals are coded by the words in $\{\mathbf{0}, \ldots, \mathbf{s}-\mathbf{1}\}^{*}$. Recall that the basic interval of $\mathfrak{F}$ generated by a word $\xi_{0} \cdots \xi_{n-1} \in\{\mathbf{0}, \ldots, \mathbf{s}-\mathbf{1}\}^{n}$ is by definition $\llbracket \xi_{0} \cdots \xi_{n-1} \rrbracket:=\hat{\mathbb{R}}_{\xi_{0} \cdots \xi_{n-1}}[0,1[$; then one has successively:

$$
\begin{aligned}
& \hat{\mathbb{R}}_{\xi_{0}}^{-1} \llbracket \xi_{0} \cdots \xi_{n-1} \rrbracket=\llbracket \xi_{1} \cdots \xi_{n-1} \rrbracket \subset[0,1], \\
& \hat{\mathbb{R}}_{\xi_{1}}^{-1} \llbracket \xi_{1} \cdots \xi_{n-1} \rrbracket=\llbracket \xi_{2} \cdots \xi_{n-1} \rrbracket \subset[0,1], \\
& \quad \vdots \\
& \hat{\mathbb{R}}_{\xi_{n-1}}^{-1} \llbracket \xi_{n-1} \rrbracket=[0,1[\subset[0,1] .
\end{aligned}
$$

Therefore, denoting by $\hat{M}_{j}:=M_{w_{j}}$, a recursive application of (12) yields:

$$
\left(\begin{array}{c}
\mu_{0} \llbracket \xi_{0} \cdots \xi_{n-1} \rrbracket \\
\vdots \\
\mu_{\mathbf{r}-1} \llbracket \xi_{0} \cdots \xi_{n-1} \rrbracket
\end{array}\right)=\hat{M}_{\xi_{0} \cdots \xi_{n-1}}\left(\begin{array}{c}
\mu_{0}[0,1[ \\
\vdots \\
\mu_{\mathbf{r}-1}[0,1[
\end{array}\right) .
$$

For $\mathscr{M}:=\left\{\hat{M}_{j}\right\}_{j=\mathbf{0}}^{\mathbf{s}-1}$, it is clear from (13) that $\mu_{0}, \ldots, \mu_{\mathbf{r}-1}$ are $\mathscr{M}$-measures w.r.t. the s-fold net $\mathfrak{F}$.

Finally, we would like to make a simple remark which may simplify the analysis of $\mu$. Let $U_{0}, \ldots, U_{\mathrm{r}-1}$ be the elements of the canonical basis of the $1 \times r$-matrix vector space. We make the third assumption that any of the matrices $\hat{M}_{j}(j=\mathbf{0}, \ldots, \mathbf{s}-\mathbf{1})$ leaves the vector space generated by $U_{k_{1}}, \ldots, U_{k_{t}}$, invariant; we denote by $\hat{M}_{j}^{\prime}(j=\mathbf{0}, \ldots, \mathbf{s}-\mathbf{1})$ the corresponding $t$-dimensional submatrices. Then (13) can be reduced to

$$
\left(\begin{array}{c}
\mu_{k_{1}} \llbracket \xi_{0} \cdots \xi_{n-1} \rrbracket \\
\vdots \\
\mu_{k_{t}} \llbracket \xi_{0} \cdots \xi_{n-1} \rrbracket
\end{array}\right)=\hat{M}_{\xi_{0} \cdots \xi_{n-1}}^{\prime}\left(\begin{array}{c}
\mu_{k_{1}}[0,1[ \\
\vdots \\
\mu_{k_{t}}[0,1[
\end{array}\right) .
$$



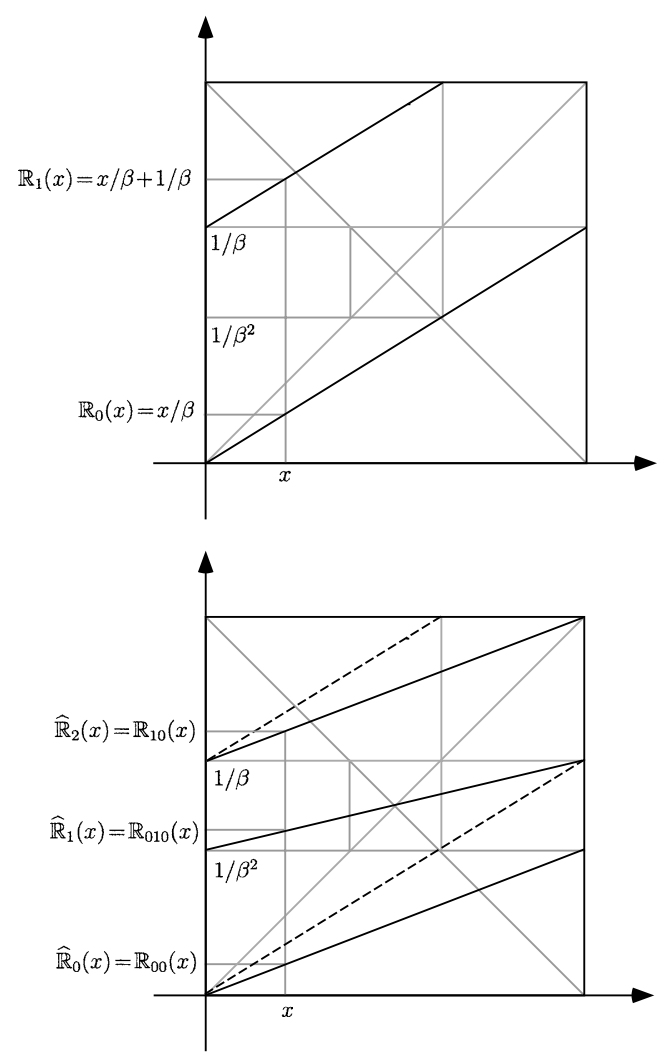

Figure 4. The first plot contains the two affine contractions $\mathbb{R}_{0}$ and $\mathbb{R}_{1}$ associated with the $\beta$-shift in the case when $\beta=(1+\sqrt{5}) / 2$. In the second plot we give an example of a s.a.c. $\mathscr{R}=\left\{\hat{\mathbb{R}}_{k}\right\}_{k=0}^{3}$ which is adapted to the unit interval

Let us introduce an auxiliary measure which we denote by $\mu_{*}$. It is associated to the Bernoulli convolution $\mu$ by putting, for any Borel subset $B$ of the real line:

$$
\mu_{*}(B)=\frac{\sum_{j=1}^{t} \mu_{k_{j}}(B)}{\sum_{j=1}^{t} \mu_{k_{j}}[0,1[},
$$

so that, for any word $w \in\{\mathbf{0}, \ldots, \mathbf{s}-1\}^{*}$,

$$
\mu_{*} \llbracket w \rrbracket:=L \hat{M}_{w}^{\prime} R,
$$

where

$$
L:=(1 \cdots 1) \quad \text { and } \quad R:=\frac{1}{\sum_{j} \mu_{k_{j}}[0,1[}\left(\begin{array}{c}
\mu_{k_{1}}[0,1[ \\
\vdots \\
\mu_{k_{t}}[0,1[
\end{array}\right) .
$$

In general, the Bernoulli convolution $\mu$ itself needs not be an $\mathscr{M}$-measure, which is why one of the main advantages of introducing successively the measures $\mu_{k_{1}}, \ldots, \mu_{k_{t}}$ and $\mu_{*}$ is the fact that they all are $\mathscr{M}$-measures. The measure $\mu_{*}$ 
proves to be a better candidate for studying its Gibbs properties; roughly speaking, this is due to the fact that the left row vector in (15) has strictly positive entries. Thus, our study of the Gibbs properties of $\mu_{*}$ (and, consequently, of $\mu$ ) will be reduced to the analysis of the convergence of the $n$-step potential $\phi_{n}(n=1,2 \ldots)-$ which, in the present case, is defined for any $\xi \in\{\mathbf{0}, \ldots, \mathbf{s}-\mathbf{1}\}^{\mathbf{N}}$ by

$$
\phi_{n}(\xi):=\log \left(\frac{L \hat{M}_{\xi_{0} \cdots \xi_{n-1}}^{\prime} R}{L \hat{M}_{\xi_{1} \cdots \xi_{n-1}}^{\prime} R}\right) .
$$

As we will see, when $\mu$ is a Bernoulli convolution associated with the multinacci numbers, the measure $\mu_{*}$ displays a clear Gibbs structure which will be analyzed in detail below (Theorem 3.2) and which ensures that the multifractal formalism holds. Nevertheless, it is worth noting that the Bernoulli convolution $\mu$ itself may not be Gibbs or weak Gibbs in a rather strong sense (see Remark 1.5). In fact, the importance of the measure $\mu_{*}$ lies in the fact that in a sense it reflects the local Gibbs structure of $\mu$ (see Proposition 4.4), which proves to be sufficient for us to show that $\mu$ itself satisfies the multifractal formalism.

2.4. Example: $\beta$-shift of finite type. In this section we assume that $\mathscr{I}_{(\beta, \mathbf{d})}=\left\{\mathbf{i}_{0}, \ldots, \mathbf{i}_{\mathbf{r}-1}\right\}$, for some $\mathbf{r} \geqslant 1$. We also assume that the $\beta$-shift is of finite type, which allows us to exhibit a s.a.c naturally associated to $\beta$. Recall that the $\beta$-shift is of finite type if and only if there exists $T \geqslant 2$ and $\varepsilon_{i} \geqslant 0$, such that

$$
1=\frac{\varepsilon_{1}}{\beta^{1}}+\cdots+\frac{\varepsilon_{T}}{\beta^{T}}
$$

together with the lexicographic conditions

$$
\varepsilon_{i} \cdots \varepsilon_{T-1}\left(\varepsilon_{T}-1\right) \varepsilon_{1} \cdots \varepsilon_{i-1} \prec_{\operatorname{lex}} \varepsilon_{1} \cdots \varepsilon_{T} \quad(2 \leqslant i \leqslant T) .
$$

Let $\mathbf{s}=\sum_{i=1}^{T} \varepsilon_{i}$; each $j \in\{\mathbf{0}, \ldots, \mathbf{s}-\mathbf{1}\}$ can be written as follows:

$$
j=\varepsilon_{1}+\cdots+\varepsilon_{k-1}+\varepsilon \quad \text { with } 1 \leqslant k \leqslant T \text { and } 0 \leqslant \varepsilon \leqslant \varepsilon_{k}-1 .
$$

Put $w_{j}=\varepsilon_{1} \cdots \varepsilon_{k-1} \varepsilon$. The s.a.c. $\mathscr{R}:=\left\{\hat{\mathbb{R}}_{j}=\mathbb{R}_{w_{j}}\right\}_{j=\mathbf{0}}^{\mathbf{s}-\mathbf{1}}$ is adapted to [0,1[ because, for any $j_{\varepsilon}=\mathbf{0}, \ldots, \mathbf{s}-\mathbf{1}$, the interval $\hat{\mathbb{R}}_{j}\left[0,1\left[\right.\right.$ is bounded from below by $\frac{\varepsilon_{1}}{\beta^{1}}+\cdots+$ $\frac{\varepsilon_{k-1}}{\beta^{k-1}}+\frac{\varepsilon}{\beta^{k}}$ and has length $\beta^{-k}$. For any word $w=\xi_{0} \cdots \xi_{n-1} \in\{\mathbf{0}, \ldots, \mathbf{s}-\mathbf{1}\}^{n}$, Lemma 2.2 yields the following matrix relation:

$$
\left(\begin{array}{c}
\mu\left(\llbracket w \rrbracket+\mathrm{i}_{0}\right) \\
\vdots \\
\mu\left(\llbracket w \rrbracket+\mathrm{i}_{\mathbf{r}-1}\right)
\end{array}\right)=M_{w_{\xi_{0}}} \cdots M_{w_{\xi_{n-1}}}\left(\begin{array}{c}
\mu\left(\left[0,1\left[+\mathrm{i}_{0}\right)\right.\right. \\
\vdots \\
\mu\left(\left[0,1\left[+\mathrm{i}_{\mathbf{r}-1}\right)\right.\right.
\end{array}\right) .
$$

Consider now the family of the matrices $\mathscr{M}:=\left\{\hat{M}_{j}=M_{w_{j}}\right\}_{j=\mathbf{0}}^{\mathbf{s}-\mathbf{1}}$; according to the definition of the measures $\mu_{k}$ in (11), the identity (16) turns into

$$
\left(\begin{array}{c}
\mu_{1} \llbracket w \rrbracket \\
\vdots \\
\mu_{\mathbf{r}-1 \llbracket w \rrbracket} \llbracket w
\end{array}\right)=\hat{M}_{w}\left(\begin{array}{c}
\mu_{1}[0,1] \\
\vdots \\
\mu_{\mathbf{r}-1}[0,1]
\end{array}\right)
$$

which means that the $\mu_{k}$ are indeed $\mathscr{M}$-measures w.r.t. the $\mathscr{R}$-net. 


\section{Bernoulli Convolution in Multinacci Bases}

Let $\mu$ be the ( $p q)$-distributed ( $\beta, 2)$-Bernoulli convolution with $p, q>0$ and $\beta$ the multinacci number of degree $\mathbf{m} \geqslant 2$, i.e., the $\mathrm{PV}$ number which is defined as the appropriate root of

$$
\beta^{\mathbf{m}}=\beta^{\mathbf{m}-1}+\cdots+\beta+1 .
$$

The set $\mathscr{I}_{(\beta, 2)}$ from Definition 2.1 has precisely $\mathbf{m}+1$ elements, namely, $\mathrm{i}_{0}=\mathbf{0}$, $\mathrm{i}_{1}=\mathbf{1}$ and $\mathrm{i}_{k}=\beta^{k-1}-\left(\beta^{k-2}+\cdots+\beta^{0}\right)$ for $k=2, \ldots, \mathbf{m}$. Fix $i \in \mathscr{B}=\{\mathbf{0}, \mathbf{1}\}$; the matrix $M_{i}$ defined in (7) is the incidence matrix of the finite automaton represented in Figure 5. It has the state set $\mathscr{I}_{(\beta, 2)}$ and the labels $\mathrm{p}=\mathrm{p}_{0}$ and $\mathrm{q}=\mathrm{p}_{1}$. The arrow from the state $\mathrm{i}_{h}$ to the state $\mathrm{i}_{k}$ has label $\mathrm{p}_{j}$ if and only if $j=i+\beta \mathrm{i}_{h}-\mathrm{i}_{k}$ belongs to $\mathscr{D}=\{\mathbf{0}, \mathbf{1}\}$. Accordingly,

$$
M_{0}=\left(\begin{array}{ccccc}
\mathrm{p} & 0 & 0 & \cdots & 0 \\
0 & 0 & \mathrm{q} & \cdots & 0 \\
\vdots & \vdots & \vdots & \ddots & \vdots \\
0 & 0 & 0 & \cdots & \mathrm{q} \\
\mathrm{q} & \mathrm{p} & 0 & \cdots & 0
\end{array}\right) \quad \text { and } \quad M_{\mathbf{1}}=\left(\begin{array}{ccccc}
\mathrm{q} & \mathrm{p} & 0 & \cdots & 0 \\
0 & 0 & 0 & \cdots & 0 \\
\vdots & \vdots & \vdots & \ddots & \vdots \\
0 & 0 & 0 & \cdots & 0 \\
0 & \mathrm{q} & 0 & \cdots & 0
\end{array}\right) \text {. }
$$

3.1. The intermediate measure $\mu_{*}$. As will be shown in Theorem 4.8, there are cases for which $\mu$ cannot be $\mathfrak{F}$-weak Gibbs for any "reasonable" s-fold net $\mathfrak{F}$. However, the multifractal analysis of $\mu$ is still possible via introducing the intermediate probability measure $\mu_{*}$, which turns out to be weak Gibbs (Theorem 3.2) and equivalent to $\mu$ in a "strong" sense (specified in Proposition 4.4). The measure $\mu_{*}$ defined in (18) below, will be given by an application of the process described in Section 2.3.

To begin with, notice that the $\beta$-shift associated to the multinacci number is of finite type, whence, by the result of Section 2.4, one can find a finite family of words $\left\{w_{j}\right\}_{j}$ in the alphabet $\{\mathbf{0}, \mathbf{1}\}$ for which the system of affine contractions $\left\{\hat{\mathbb{R}}_{j}=\mathbb{R}_{w_{j}}\right\}_{j}$ is adapted to the unit interval. However, for the sake of simplicity, our family of words will be different from the one obtained by the systematic approach presented in Section 2.4. This way we obtain a family of just $2 \times 2$ matrices.
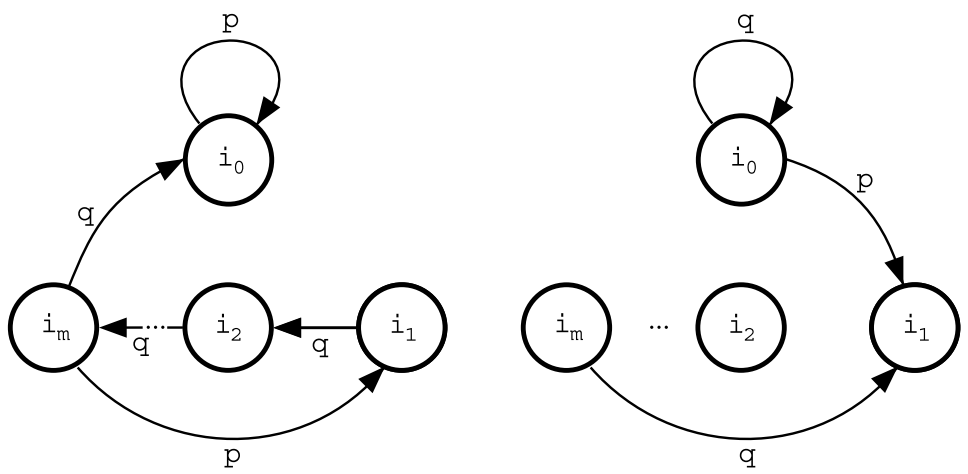

Figure 5. For $\beta$ being the multinacci number of order $\mathbf{m}$, we represent the automaton of the relation $\cdot \triangleright^{i}$. on $\mathscr{I}_{(\beta, 2)}$, for $i=\mathbf{0}$ (left) and $i=\mathbf{1}$ (right) 

by

Heuristically, our approach is based on the following two remarks. Denoting

$$
X=\left(\begin{array}{llllll}
1 & 0 & 0 & 0 & \cdots & 0 \\
0 & 0 & 1 & 0 & \cdots & 0
\end{array}\right)
$$

one obtains first

$$
X M_{\mathbf{0}^{\mathrm{m}}}=\left(\begin{array}{cc}
\mathrm{p}^{\mathbf{m}} & 0 \\
\mathrm{pq}^{\mathbf{m}-1} & \mathrm{pq}^{\mathbf{m}-1}
\end{array}\right) X
$$

Furthermore, since ${ }^{4} M_{\mathbf{0}^{q} \mathbf{1}^{r} \mathbf{1}} \prec\left(\begin{array}{ccccc}1 & 1 & 0 & \cdots & 0 \\ \vdots & \vdots & \vdots & \ddots & \vdots \\ 1 & 1 & 0 & \cdots & 0\end{array}\right)$, for any $q, r \geqslant 0$, it is also
clear that

$$
X M_{0^{q}} 1^{r} 10 \prec\left(\begin{array}{ll}
1 & 1 \\
1 & 1
\end{array}\right) X .
$$

Actually, there exists a family of words $w_{j}$ of the form either $\mathbf{0}^{\mathbf{m}}$ or $\mathbf{0}^{x} \mathbf{1}^{y} \mathbf{1 0}$, for which the system $\left\{\mathbb{R}_{w_{j}}\right\}_{j}$ is adapted to the unit interval. To see this, notice that the algebraic property of $\beta$ ensures that the semi-open intervals $\mathbb{R}_{\mathbf{1}^{y} \mathbf{1 0}}[0,1[$, for $0 \leqslant y \leqslant \mathbf{m}-2$, form a partition of $\left[1 / \beta, 1\right.$. Moreover, $\mathbb{R}_{\mathbf{0}^{\mathbf{m}}}\left[0,1\left[\right.\right.$ and $\mathbb{R}_{\mathbf{0}^{x}}[1 / \beta, 1[$, for $0 \leqslant x \leqslant \mathbf{m}-1$, form a partition of $[0,1$ [. For a suitable labelling of the words $\mathbf{0}^{x} \mathbf{1}^{y} \mathbf{1 0}$, let $j=j(x, y)$ be the integer such that

$$
j-1:=(\mathbf{m}-1)((\mathbf{m}-1)-x)+y .
$$

The uniqueness of the division of $j-1$ by $\mathbf{m}-1$ with remainder implies $(x, y) \mapsto j$ is a bijection from $\{0, \ldots, \mathbf{m}-1\} \times\{0, \ldots, \mathbf{m}-2\}$ onto $\{1, \ldots, \mathbf{m}(\mathbf{m}-1)\}$. Next, we consider the adapted system $\mathscr{R}:=\left\{\hat{\mathbb{R}}_{j}=\mathbb{R}_{w_{j}}\right\}_{j=0}^{\mathbf{m}(\mathbf{m}-1)}$, where

$$
w_{0}:=\mathbf{0}^{\mathbf{m}} \text { and } w_{j}:=\mathbf{0}^{\mathbf{x}(j)} \mathbf{1}^{\mathbf{y}(j)} \mathbf{1 0} \text { with }\left\{\begin{array}{l}
\mathbf{x}(j):=(\mathbf{m}-1)-\left[\frac{j-1}{\mathbf{m}-1}\right] \\
\mathbf{y}(j):=(\mathbf{m}-1)\left\{\frac{j-1}{\mathbf{m}-1}\right\}
\end{array}\right.
$$

(here, $[\cdot]$ and $\{\cdot\}$ stand respectively for the integral and the fractional part of a

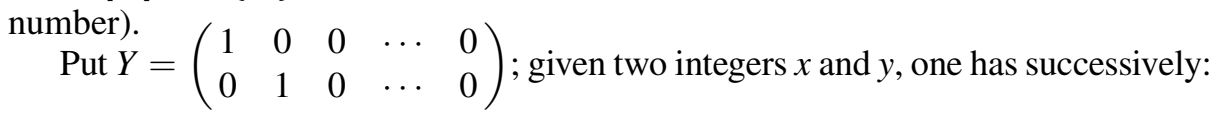

$$
X M_{\mathbf{0}^{x}}=\left\{\begin{array}{cc}
\left(\begin{array}{cc}
\mathrm{p}^{x} \mathrm{q} & \mathrm{p}^{x+1} \\
0 & 0
\end{array}\right) Y & \text { if } x<\mathbf{m}-2 \\
\left(\begin{array}{cc}
\mathrm{p}^{\mathbf{m}-\mathbf{2}} \mathrm{q} & \mathrm{p}^{\mathbf{m}-\mathbf{1}} \\
0 & \mathrm{q}^{\mathbf{m}-1}
\end{array}\right) Y & \text { if } x=\mathbf{m}-2 \\
\left(\begin{array}{cc}
\mathrm{p}^{\mathbf{m}-1} \mathrm{q} & \mathrm{p}^{\mathbf{m}} \\
\mathrm{q}^{\mathbf{m}} & \mathrm{q}^{\mathbf{m}-1} \mathrm{p}
\end{array}\right) Y & \text { if } x=\mathbf{m}-1
\end{array}\right.
$$

\footnotetext{
${ }^{4}$ Given two square matrices $A=\left(a_{i, j}\right)$ and $B=\left(b_{i, j}\right)$ with nonnegative entries, we write $A \prec B$ whenever $b_{i, j}=0$ implies that $a_{i, j}=0$, for any $i, j$.
} 
and

$$
Y M_{\mathbf{1}^{y} \mathbf{0}}= \begin{cases}\left(\begin{array}{cc}
\mathrm{p} & 0 \\
0 & \mathrm{q}
\end{array}\right) X & \text { if } y=0 \\
\left(\begin{array}{cc}
\mathrm{q}^{y} \mathrm{p} & \mathrm{q}^{y} \mathrm{p} \\
0 & 0
\end{array}\right) X & \text { if } y>0 .\end{cases}
$$

Put $\alpha=(\mathbf{q} / \mathbf{p})^{\mathbf{m}-1}$; a straightforward computation yields the following

Lemma 3.1. For any $j \in \mathscr{J}:=\{\mathbf{0}, \ldots, \mathbf{m}(\mathbf{m}-1)\}$ one has $X M_{w_{j}}=P_{j} X$, where

- $P_{\mathbf{0}}:=\mathrm{p}^{\mathbf{m}}\left(\begin{array}{cc}1 & 0 \\ \alpha & \alpha\end{array}\right)$ when $j=\mathbf{0}$ (i.e., $\left.w_{j}=\mathbf{0}^{\mathbf{m}}\right)$;

- $P_{j}:=\mathrm{p}^{\mathbf{m}} \mathrm{q}^{j}\left(\begin{array}{cc}1 & 1 \\ \alpha & \alpha\end{array}\right)$ when $\mathbf{0}<j<\mathbf{m}$ (i.e., $\left.w_{j}=\mathbf{0}^{\mathbf{m}-1} \mathbf{1}^{j-1} \mathbf{1 0}\right)$;

- $P_{\mathbf{m}}:=\mathrm{q}^{\mathbf{m}}\left(\begin{array}{cc}1 / \alpha & 1 / \alpha \\ 0 & 1\end{array}\right)$ when $j=\mathbf{m}$ (i.e., $\left.w_{j}=\mathbf{0}^{\mathbf{m}-2} \mathbf{1 0}\right)$;

- $P_{j}:=\mathrm{p}^{\mathrm{x}(j)+1} \mathrm{q}^{\mathrm{y}(j)+1}\left(\begin{array}{ll}1 & 1 \\ 0 & 0\end{array}\right)$ when $\mathbf{m}<j \leqslant \mathbf{m}(\mathbf{m}-1)$.

From here on, we will consider the basic intervals associated to the adapted system $\mathscr{R}$. For any word $w \in \mathscr{J}^{*}$ we denote $\llbracket w \rrbracket=\hat{\mathbb{R}}_{w}[0 ; 1[$; by (14) and Lemma 3.1,

$$
\left(\begin{array}{c}
\mu_{0} \llbracket w \rrbracket \\
\mu_{2} \llbracket w \rrbracket
\end{array}\right)=P_{w}\left(\begin{array}{c}
\mu_{0}([0,1]) \\
\mu_{2}([0,1])
\end{array}\right) .
$$

(Recall that $\mu_{i}(\cdot):=\mu\left(\cdot \cap[0,1]+\mathbf{i}_{i}\right)$.) We introduce the probability

$$
\mu_{*}:=\frac{1}{\mu_{0}([0,1])+\mu_{2}([0,1])}\left(\mu_{0}+\mu_{2}\right)
$$

so that, for any word $w \in \mathscr{J}^{*}$,

$$
\mu_{*} \llbracket w \rrbracket=L P_{w} R,
$$

where

$$
L=\left(\begin{array}{ll}
1 & 1
\end{array}\right) \quad \text { and } \quad R=\frac{1}{\mu_{0}([0,1])+\mu_{2}([0,1])}\left(\begin{array}{c}
\mu_{0}([0,1]) \\
\mu_{2}([0,1])
\end{array}\right)=\left(\begin{array}{c}
1-q^{\mathbf{m}-1} \\
q^{\mathbf{m}-1}
\end{array}\right) .
$$

Now we are ready to formulate one of the central claims of the present paper:

Theorem 3.2. The Gibbs properties of $\mu_{*}$ w.r.t. the net $\mathfrak{F}$ associated to the adapted system $\mathscr{R}$, are the following:

(i) when $\mathbf{m}=2$, the measure $\mu_{*}$ is weak Gibbs if $\mathrm{p}=\mathrm{q}$ and Gibbs if $\mathrm{p} \neq \mathrm{q}$;

(ii) when $\mathbf{m} \geqslant 3$, the measure $\mu_{*}$ is weak Gibbs if $\mathbf{p}=\mathbf{q}$ and Gibbs if $\mathbf{p}>\mathbf{q}$.

Remark 3.3. The measure $\mu_{*}$ is not even $\mathfrak{F}$-weak Gibbs when $\mathbf{m} \geqslant 3$ and $\mathbf{p}<\mathbf{q}$ : consider the basic interval $\llbracket \xi_{0} \cdots \xi_{2 n-1} \rrbracket=\llbracket \mathbf{m}^{n}(\mathbf{m}+1)^{n} \rrbracket$; then, it is clear that the ratio

$$
\frac{\mu * \llbracket \xi_{0} \cdots \xi_{2 n-1} \rrbracket}{\mu_{*} \llbracket \xi_{0} \cdots \xi_{n-1} \rrbracket \mu_{*} \llbracket \xi_{n} \cdots \xi_{2 n-1} \rrbracket}
$$


does not satisfy the condition

$$
\frac{1}{K_{n}} \leqslant \frac{\mu_{*} \llbracket \xi_{0} \cdots \xi_{2 n-1} \rrbracket}{\mu_{*} \llbracket \xi_{0} \cdots \xi_{n-1} \rrbracket \mu_{*} \llbracket \xi_{n} \cdots \xi_{2 n-1} \rrbracket} \leqslant K_{n}
$$

together with $\lim _{n} \frac{1}{n} \log K_{n}=0$ (this will be explained in more detail in Remark 3.5). In the case $\mathbf{m} \geqslant 3$ and $p<q$, the problem of the existence of a net $\mathfrak{F}^{\prime}$ of $[0,1]$, w.r.t. which $\mu_{*}$ is $\mathfrak{F}^{\prime}$-weak Gibbs, remains open.

Theorem 3.2 is a corollary of Theorem 3.4 below, whose formulation needs the explicit expression for a potential $\Phi$ associated with $\mu_{*}$.

3.2. The potential associated to $\mu_{*}$. In order to compute the limit potential of the $n$-step potential associated to the measure $\mu_{*}$, we need some classical facts about continued fractions which can be found in [24]. Let $u_{0}, u_{1}, \ldots$ and $v_{0}, v_{1}, \ldots$ be two infinite sequences of real numbers, both assumed to be positive, except, possibly, $u_{0}$ which is allowed to be nonnegative. For any $n \geqslant 0$ the positive reals $p_{n}$ and $q_{n}$ are defined by

$$
\left(\begin{array}{l}
p_{n} \\
q_{n}
\end{array}\right)=\left(\begin{array}{cc}
u_{0} & v_{0} \\
1 & 0
\end{array}\right)\left(\begin{array}{cc}
u_{1} & v_{1} \\
1 & 0
\end{array}\right) \cdots\left(\begin{array}{cc}
u_{n} & v_{n} \\
1 & 0
\end{array}\right)\left(\begin{array}{l}
1 \\
0
\end{array}\right)
$$

and by convention, $\left(\begin{array}{l}p_{-1} \\ q_{-1}\end{array}\right)=\left(\begin{array}{l}1 \\ 0\end{array}\right)$. Then $p_{n} / q_{n}$ is the continued fraction associated with $u_{0}, \ldots, u_{n}$ and $v_{0}, \ldots, v_{n}$ in the usual sense: for any $n \geqslant 0$,

$$
\frac{p_{n}}{q_{n}}=u_{0}+\frac{v_{0}}{u_{1}+\frac{v_{1}}{\ddots u_{n-1}+\frac{v_{n-1}}{u_{n}}}}
$$

(see [24] for the proof). A direct consequence of (19) is the following relation:

$$
\left(\begin{array}{cc}
u_{0} & v_{0} \\
1 & 0
\end{array}\right) \cdots\left(\begin{array}{cc}
u_{n} & v_{n} \\
1 & 0
\end{array}\right)=\left(\begin{array}{cc}
p_{n} & v_{n} p_{n-1} \\
q_{n} & v_{n} q_{n-1}
\end{array}\right) .
$$

Also, for $n \geqslant 1$, we have

$$
\left\{\begin{array}{l}
p_{n}=u_{n} p_{n-1}+v_{n-1} p_{n-2} \\
q_{n}=u_{n} q_{n-1}+v_{n-1} q_{n-2} .
\end{array}\right.
$$

Consider a special case of such continued fractions. Recall that $\alpha=(\mathbf{q} / \mathrm{p})^{\mathbf{m}-1}$; given a sequence of integers $a_{0}, a_{1}, \ldots$ with $a_{0} \geqslant 0$ and $a_{i}>0$ for $i \geqslant 1$ and $\kappa=\mathbf{0}$ or $\mathbf{1}$, put

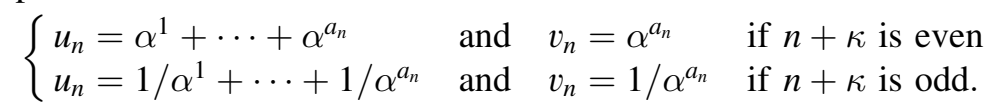

By convention, put $u_{0}=0$ in either case when $a_{0}=0$. Then the sequences $\left(p_{n}\right)_{n=-1}^{\infty}$ and $\left(q_{n}\right)_{n=-1}^{\infty}$ are defined by (19). The sequence $\left(p_{2 n} / q_{2 n}\right)_{n=0}^{\infty}$ is nondecreasing while $\left(p_{2 n+1} / q_{2 n+1}\right)_{n=0}^{\infty}$ is nonincreasing, and $p_{2 n} / q_{2 n} \leqslant p_{2 n+1} / q_{2 n+1}$. Moreover, setting $\rho:=\min \{\sqrt{\alpha}, 1 / \sqrt{\alpha}\}$, for any $n \geqslant 1$, one has

$$
\left|\frac{p_{n}}{q_{n}}-\frac{p_{n-1}}{q_{n-1}}\right| \leqslant \begin{cases}\frac{\rho^{a_{1}+\cdots+a_{n}}}{\rho^{2+2 a_{0}+a_{n}}} & \text { if } \alpha \neq 1 \\ \frac{1}{a_{0}+\cdots+a_{n}} & \text { if } \alpha=1 .\end{cases}
$$


This is a property of the regular continued fractions when $\alpha=1$ (see, e.g., [15]) and corresponds to part (ii) of Lemma 3.8 in case $\alpha \neq 1$, see below. Analogously to the regular continued fractions, we denote

$$
\left[\kappa \mid a_{0} ; a_{1}, \ldots, a_{n}\right]=\frac{p_{n}}{q_{n}} \quad \text { and }\left\{\begin{array}{l}
{\left[\kappa \mid a_{0} ; a_{1}, \ldots, a_{n}, \infty\right]=\lim _{k \rightarrow \infty}\left[\kappa \mid a_{0} ; a_{1}, \ldots, a_{n}, k\right]} \\
{\left[\kappa \mid a_{0} ; a_{1}, a_{2}, \ldots\right]=\lim _{n \rightarrow \infty}\left[\kappa \mid a_{0} ; a_{1}, \ldots, a_{n}\right] .}
\end{array}\right.
$$

We also define the matrix

$$
Q_{\kappa}\left(a_{0}, \ldots, a_{n}\right)=\left(\begin{array}{cc}
u_{0} & v_{0} \\
1 & 0
\end{array}\right) \cdots\left(\begin{array}{cc}
u_{n} & v_{n} \\
1 & 0
\end{array}\right)
$$

and, for any column vector $\left(\begin{array}{l}x \\ y\end{array}\right)$ with nonnegative entries,

$\left[\kappa\left|a_{0} ; a_{1}, \ldots, a_{n}\right|\left(\begin{array}{l}x \\ y\end{array}\right)\right]=\frac{p_{n}(x, y)}{q_{n}(x, y)}$ with $\left(\begin{array}{l}p_{n}(x, y) \\ q_{n}(x, y)\end{array}\right)=Q_{\kappa}\left(a_{0}, \ldots, a_{n}\right)\left(\begin{array}{l}x \\ y\end{array}\right)$.

Notice that if $\Delta$ denotes $\left(\begin{array}{ll}0 & 1 \\ 1 & 0\end{array}\right)$, then for any $n \geqslant 0$,

$$
\left(\begin{array}{cc}
u_{n} & v_{n} \\
1 & 0
\end{array}\right)= \begin{cases}\Delta\left(\frac{1}{\mathrm{p}^{\mathbf{m}}} P_{\mathbf{0}}\right)^{a_{n}}, & \text { if } n+\kappa \text { is even and } \\
\left(\frac{1}{\mathbf{q}^{\mathbf{m}}} P_{\mathbf{m}}\right)^{a_{n}} \Delta, & \text { if } n+\kappa \text { is odd. }\end{cases}
$$

This allows to compute the potential $\Phi: \mathscr{J}^{\mathbf{N}} \rightarrow \mathbf{R}$ associated with $\mu_{*}$. To do so, let us first introduce a suitable notation for the words in $\{\mathbf{0}, \mathbf{m}\}^{*}$ : given $\xi=\mathbf{0}$ or $\mathbf{m}$, we denote by $\langle\xi \mid a\rangle$ the word $\xi^{a}$, for any nonnegative integer $a$. Furthermore, for any sequence of nonnegative integers $a_{1}, \ldots, a_{n}(n \geqslant 2)$, we define the word $\left\langle\xi \mid a_{1}, \ldots, a_{n}\right\rangle$ by the induction relation

$$
\left\langle\xi \mid a_{1}, \ldots, a_{n}\right\rangle=\left\langle\xi \mid a_{1}\right\rangle\left\langle\mathbf{m}-\xi \mid a_{2}, \ldots, a_{n}\right\rangle .
$$

Before proving the uniform convergence of the $n$-step potential associated with $\mu_{*}$, we give an explicit formula for the limit potential which we denote by $\Phi$. Given $j \in \mathscr{J}$, we distinguish between the same cases as in Lemma 3.1. Put

$$
\text { - } \Xi(j):= \begin{cases}\mathrm{p}^{\mathbf{m}} \alpha^{2} & \text { if } j=\mathbf{0} ; \\ \mathrm{p}^{\mathbf{m}} \mathrm{q}^{j}(1+\alpha) & \text { if } \mathbf{0}<j<\mathbf{m} ; \\ \mathrm{q}^{\mathbf{m}} / \alpha^{2} & \text { if } j=\mathbf{m} ; \\ \mathrm{p}^{\mathrm{x}(j)+1} \mathrm{q}^{\mathrm{y}(j)+1} & \text { if } \mathbf{m}<j \leqslant \mathbf{m}(\mathbf{m}-1)\end{cases}
$$

and, if $j \in \mathscr{J} \backslash\{\mathbf{0}, \mathbf{m}\}$,

$$
\text { - } X_{j}:= \begin{cases}\left(\begin{array}{l}
1 \\
\alpha
\end{array}\right) & \text { if } \mathbf{0}<j<\mathbf{m} \\
\left(\begin{array}{l}
1 \\
0
\end{array}\right) & \text { if } \mathbf{m}<j \leqslant \mathbf{m}(\mathbf{m}-1) .\end{cases}
$$

Given $\kappa \in\{\mathbf{0}, \mathbf{1}\}$, we denote $\hat{\kappa}=\mathbf{1}-\kappa$ and $\kappa \star \mathbf{m}$ stands for either $\mathbf{0}$ or $\mathbf{m}$ when $\kappa$ is either $\mathbf{0}$ or $\mathbf{1}$ respectively; hence, $\kappa \star \mathbf{m}$ is just "multiplication" of $\kappa$ by $\mathbf{m}$, which is not be confused with the concatenation $\kappa \mathbf{m}$. 
Let $\mathscr{J}^{\mathbf{N}} \ni \xi=w j \omega$, with $\{\mathbf{0}, \mathbf{m}\}^{*} \ni w=\left\langle\kappa \star \mathbf{m} \mid a_{1}, \ldots, a_{n}\right\rangle, j \in \mathscr{J} \backslash\{\mathbf{0}, \mathbf{m}\}$ and $\omega \in \mathscr{J}^{\mathbf{N}}$. Assume $\kappa=\mathbf{0}$ (the case $\kappa=\mathbf{1}$ is symmetric); for $k \geqslant 1+a_{1}+\cdots+a_{n}$ direct computation yields

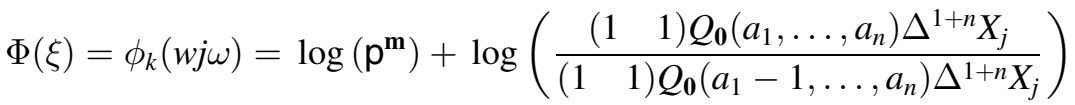

$$
\begin{aligned}
& =\log \Xi(\mathbf{0})+\log \left(\frac{\left(\begin{array}{ll}
1 & 0
\end{array}\right) Q_{\mathbf{1}}\left(1, a_{1}, \ldots, a_{n}\right) \Delta^{1+n} X_{j}}{\left(\begin{array}{ll}
0 & 1
\end{array}\right) Q_{\mathbf{1}}\left(1, a_{1}, \ldots, a_{n}\right) \Delta^{1+n} X_{j}}\right) .
\end{aligned}
$$

More generally, for $\kappa \in\{\mathbf{0}, \mathbf{1}\}, j \in \mathscr{J} \backslash\{\mathbf{0}, \mathbf{m}\}$ and $\omega \in \mathscr{J}^{\mathbf{N}}$,

- $\Phi(w j \omega)=\log \left(\Xi(\kappa \star \mathbf{m})\left[\hat{\kappa}\left|1 ; a_{1}, \ldots, a_{n}\right| \Delta^{\hat{\kappa}+n} X_{i}\right]\right)$ if $w=\left\langle\kappa \star \mathbf{m} \mid a_{1}, \ldots, a_{n}\right\rangle$;

- $\Phi(j \omega)=\log (\Xi(j))$;

- $\Phi(\omega)= \begin{cases}\log \left(\Xi(\kappa \star \mathbf{m})\left[\hat{\kappa} \mid 1 ; a_{1}, \ldots, a_{n}, \infty\right]\right) & \text { if } \omega=\left\langle\kappa \star \mathbf{m} \mid a_{1}, \ldots, a_{n}, \infty\right\rangle \\ \log \left(\Xi(\kappa \star \mathbf{m})\left[\hat{\kappa} \mid 1 ; a_{1}, a_{2}, \ldots\right]\right) & \text { if } \omega=\left\langle\kappa \star \mathbf{m} \mid a_{1}, a_{2}, \ldots\right\rangle .\end{cases}$

We have sketched the proof of the pointwise convergence of the $n$-step potential $\phi_{k}$ to the potential $\Phi$ whose expression is given above. Indeed, by (24), this is a simple consequence of the convergence of the continued fractions involved. We need however to show that the convergence is uniform; this is dealt with in the following theorem, by means of considering the two alternative cases $\mathrm{p} \neq \mathrm{q}$ and $\mathrm{p}=\mathrm{q}$.

Theorem 3.4. Let $\rho:=\min \{\sqrt{\alpha}, 1 / \sqrt{\alpha}\} \leqslant 1$; there exists a constant $K>0$ such that, for any $\omega \in \mathscr{J}^{\mathbf{N}}$ and any $n \geqslant 1$, we have

$$
\left\|\phi_{n}-\Phi\right\|_{\infty} \leqslant \begin{cases}K \rho^{n} & \text { if } \mathrm{p}>\mathrm{q} \\ K \rho^{n} & \text { if } \mathrm{p} \neq \mathrm{q} \text { and } \mathbf{m}=2 \\ K / n & \text { if } \mathrm{p}=\mathrm{q} .\end{cases}
$$

Remark 3.5. The potential $\Phi$ is continuous except when $\mathrm{p}<\mathrm{q}$ and $\mathbf{m} \geqslant 3$, as in that case $\Phi$ is discontinuous at $\omega=\mathbf{m}^{\infty}$. Indeed, as we have already noticed (see Remark 3.3), $\mu_{*}$ is not $\mathfrak{F}$-weak Gibbs if $\mathrm{p}<\mathrm{q}$ and $\mathbf{m} \geqslant 3$. Let us also point out that $\Phi$ is Hölder continuous if either $\mathbf{p}>\mathbf{q}$ or $\mathbf{p} \neq \mathbf{q}$ and $\mathbf{m}=2$, yielding $\mu_{*}$ to be $\mathfrak{F}$-Gibbs in these cases.

3.3. Preliminary to proof of Theorem 3.4. The key argument is based on the fact that the pointwise convergence of the $n$-step potential to a continuous limit implies the uniform convergence. To see this, let us define the $n$-step variation of an arbitrary map $f: \Omega:=\mathscr{A}^{\mathbf{N}} \rightarrow \mathbf{R}$ with $\mathscr{A}:=\{\mathbf{0}, \ldots, \mathbf{s}-\mathbf{1}\}$, by defining

$$
\operatorname{Var}_{n}(f)=\sup \left\{f(\xi)-f\left(\xi^{0}\right) ;\left(\xi, \xi^{0}\right) \in \mathscr{A}^{\mathbf{N}} \times \mathscr{A}^{\mathbf{N}} \text { and } \xi_{0} \cdots \xi_{n-1}=\xi_{0}^{0} \cdots \xi_{n-1}^{0}\right\} .
$$

It is clear that $f$ is continuous if and only if $\lim _{n \rightarrow \infty} \operatorname{Var}_{n}(f)=0$.

Lemma 3.6. Suppose that $\mathscr{S}$ is an s.a.c. adapted to the interval $[0,1]$ and let $\eta$ be a probability measure whose support is a subset of $[0,1]$. If the $n$-step potential $\phi_{n}$ associated to $\eta$ converges to $\phi: \Omega \rightarrow \mathbf{R}$ pointwise, then $\left\|\phi_{n}-\phi\right\|_{\infty} \leqslant \operatorname{Var}_{n}(\phi)$.

Proof. Given an arbitrary rank $n$ and any $\varepsilon>0$, the pointwise convergence of $\phi_{n}$ to $\phi$ implies that for any $\xi \in \Omega$, there exists an integer $N(\xi) \geqslant n$ such that

$$
\left|\phi_{N(\xi)}(\xi)-\phi(\xi)\right| \leqslant \varepsilon \text {. }
$$


Since the product space $\Omega$ is compact, there exists a finite set $X \subset \Omega$ such that

$$
\Omega=\bigcup_{\xi \in X} \llbracket \xi_{0} \cdots \xi_{N(\xi)-1} \rrbracket .
$$

In the product space $\Omega$, the intersection of any pair of cylinders is either empty or coincides with one of them, whence we may take a set smaller than $X$ so that the union in (28) becomes disjoint. For any $\omega \in \Omega$ put $X_{\omega, n}=X \cap\left[\omega_{0} \cdots \omega_{n-1}\right]$. Then

whence

$$
\exp \left(\phi_{n}(\omega)\right)=\frac{\eta \llbracket \omega_{0} \cdots \omega_{n-1} \rrbracket}{\eta \llbracket \omega_{1} \cdots \omega_{n-1} \rrbracket}=\frac{\sum_{\xi \in X_{\omega, n}} \eta \llbracket \xi_{0} \cdots \xi_{N(\xi)-1} \rrbracket}{\sum_{\xi \in X_{\omega, n}} \eta \llbracket \xi_{1} \cdots \xi_{N(\xi)-1} \rrbracket}
$$

$$
\min _{\xi \in X_{\omega, n}}\left\{\frac{\eta \llbracket \xi_{0} \cdots \xi_{N(\xi)-1} \rrbracket}{\eta \llbracket \xi_{1} \cdots \xi_{N(\xi)-1} \rrbracket}\right\} \leqslant \exp \left(\phi_{n}(\omega)\right) \leqslant \max _{\xi \in X_{\omega, n}}\left\{\frac{\eta \llbracket \xi_{0} \cdots \xi_{N(\xi)-1} \rrbracket}{\eta \llbracket \xi_{1} \cdots \xi_{N(\xi)-1} \rrbracket}\right\} .
$$

Since by definition

$$
\frac{\eta \llbracket \xi_{0} \cdots \xi_{N(\xi)-1} \rrbracket}{\eta \llbracket \xi_{1} \cdots \xi_{N(\xi)-1} \rrbracket}=\exp \left(\phi_{N(\xi)}(\xi)\right),
$$

we obtain, in view of (27),

$$
\min _{\xi \in X_{\omega, n}}\{\phi(\xi)\}-\varepsilon \leqslant \phi_{n}(\omega) \leqslant \max _{\xi \in X_{\omega, n}}\{\phi(\xi)\}+\varepsilon
$$

and thus,

$$
\phi(\omega)-\operatorname{Var}_{n}(\phi)-\varepsilon \leqslant \phi_{n}(\omega) \leqslant \phi(\omega)+\operatorname{Var}_{n}(\phi)+\varepsilon .
$$

Since (29) holds for an arbitrary $\varepsilon>0$, we have $\left|\phi_{n}(\omega)-\phi(\omega)\right| \leqslant \operatorname{Var}_{n}(\phi)$, which concludes the proof.

In what follows, the sequences $\left(u_{n}\right)_{n=0}^{\infty},\left(v_{n}\right)_{n=0}^{\infty}, \ldots$ are always associated with $\kappa \in\{\boldsymbol{0}, \mathbf{1}\}$ and $a_{0}, a_{1}, \ldots$, as in (23), (19) and (25). For any $n \geqslant 1$, we introduce the following quantity:

$$
\delta_{n}:=\left|\frac{p_{n}}{q_{n}}-\frac{p_{n-1}}{q_{n-1}}\right| .
$$

We need two lemmas which involve $\delta_{n}$.

Lemma 3.7. For any integer $n \geqslant 1$ and any column vector with nonnegative entries $\left(\begin{array}{l}x \\ y\end{array}\right) \neq\left(\begin{array}{l}0 \\ 0\end{array}\right)$, one has:

(i) $\left|\frac{p_{n}(x, y)}{q_{n}(x, y)}-\frac{p_{n}}{q_{n}}\right| \leqslant \frac{y v_{n}}{x u_{n}+y v_{n}} \cdot \delta_{n}$ and

(ii) $\delta_{n+1} \leqslant \frac{v_{n}}{u_{n} u_{n+1}+v_{n}} \cdot \delta_{n}$.

Proof. (i) By (21),

$$
\begin{aligned}
\frac{p_{n}(x, y)}{q_{n}(x, y)} & =\frac{\left(\begin{array}{ll}
1 & 0
\end{array}\right)\left(\begin{array}{ll}
p_{n} & v_{n} p_{n-1} \\
q_{n} & v_{n} q_{n-1}
\end{array}\right)\left(\begin{array}{l}
x \\
y
\end{array}\right)}{\left(\begin{array}{ll}
0 & 1
\end{array}\right)\left(\begin{array}{ll}
p_{n} & v_{n} p_{n-1} \\
q_{n} & v_{n} q_{n-1}
\end{array}\right)\left(\begin{array}{l}
x \\
y
\end{array}\right)}=\frac{x p_{n}+y v_{n} p_{n-1}}{x q_{n}+y v_{n} q_{n-1}} \\
& =\frac{a q_{n}}{x q_{n}+y v_{n} q_{n-1}} \cdot \frac{p_{n}}{q_{n}}+\frac{y v_{n} q_{n-1}}{x q_{n}+y v_{n} q_{n-1}} \cdot \frac{p_{n-1}}{q_{n-1}}
\end{aligned}
$$


whence

$$
\frac{p_{n}(x, y)}{q_{n}(x, y)}-\frac{p_{n}}{q_{n}}=\frac{y v_{n} q_{n-1}}{x q_{n}+y v_{n} q_{n-1}} \cdot\left(\frac{p_{n-1}}{q_{n-1}}-\frac{p_{n}}{q_{n}}\right)
$$

and since $q_{n} \geqslant u_{n} q_{n-1}$, we are done.

(ii) We obtain (ii) by simply applying (i) to the vector $\left(\begin{array}{l}x \\ y\end{array}\right)=\left(\begin{array}{c}u_{n+1} \\ 1\end{array}\right)$.

Lemma 3.8. (i) For an arbitrary rank $n \geqslant 1$ we have $\delta_{n+1} \leqslant \delta_{n}$, and

$$
\delta_{n} \leqslant \frac{\rho^{a_{1}+\cdots+a_{n}}}{\rho^{2+2 a_{0}}}
$$

in either of the following cases: $n+\kappa$ is even and $\alpha>1$; or $n+\kappa$ is odd and $\alpha<1$;

(ii) if $\alpha \neq 1$, then for any $n \geqslant 1$,

$$
\delta_{n} \leqslant \frac{\rho^{a_{1}+\cdots+a_{n}}}{\rho^{2+2 a_{0}+a_{n}}}
$$

(iii) if $\alpha \neq 1$, then there exists a constant $K>0$ such that, for arbitrary rank $n \geqslant 1$ and any integer $0<a<a_{n}$,

$$
\left|\left[\kappa \mid a_{0} ; a_{1}, \ldots, a_{n-1}, a_{n}\right]-\left[\kappa \mid a_{0} ; a_{1}, \ldots, a_{n-1}, a\right]\right| \leqslant \frac{K}{\rho^{2 a_{0}}} \rho^{a_{1}+\cdots+a_{n-1}+a} .
$$

Proof. (i) We are going to establish the inequality in (i) in the case when $n+\kappa$ is even with $\alpha>1$; this implies the inequality in the opposite case, as the value of $\delta_{n}$ remains the same whenever a pair $(\alpha, \kappa)$ is replaced by $\left(\alpha^{\prime}=1 / \alpha>1, \hat{\kappa}=1-\kappa\right)$.

Assume now $n+\kappa$ is even, and let $k \leqslant n$ be such that $k+\kappa$ is even as well. Then, on one hand, Lemma 3.7 (ii) implies

$$
\frac{\delta_{k}}{\delta_{k-1}} \leqslant \frac{v_{k-1}}{u_{k-1} u_{k}} \leqslant \frac{1 / \alpha^{a_{k-1}}}{(1 / \alpha) \alpha^{a_{k}}} \leqslant \frac{1}{\alpha^{\left(a_{k}+a_{k-1}\right) / 2}},
$$

and on the other hand, since $\delta_{k-1} / \delta_{k-2} \leqslant 1$,

$$
\delta_{n} \leqslant \frac{\delta_{n}}{\delta_{n-1}} \frac{\delta_{n-2}}{\delta_{n-3}} \cdots \frac{\delta_{\kappa+2}}{\delta_{\kappa+1}} \delta_{1} \leqslant \frac{\delta_{1}}{\alpha^{\left(a_{n}+a_{n-1}+\cdots+a_{\kappa+1}\right) / 2}} .
$$

This proves (i), as $\delta_{1}=v_{0} / u_{1}$ is bounded by $\alpha^{1+a_{0}}$ if $\kappa=0$ and by $1 / \alpha^{a_{0}+a_{1}} \leqslant \alpha^{1+a_{0}} / \alpha^{a_{1} / 2}$ if $\kappa=1$.

(ii) and (iii) Part (ii) is a straightforward consequence of (i) and the definition of $\rho$. In order to prove (iii), we consider, for any integer $0<a<a^{\prime}$, the quantity

$$
\delta_{n}\left(a, a^{\prime}\right):=\left|\left[\kappa \mid a_{0} ; a_{1}, \ldots, a_{n-1}, a^{\prime}\right]-\left[\kappa \mid a_{0} ; a_{1}, \ldots, a_{n-1}, a\right]\right| \leqslant \sum_{i=a}^{a^{\prime}-1} \delta_{n}(i, i+1) .
$$

Since $\left[\kappa \mid a_{0} ; a_{1}, \ldots, a_{n-1}, i+1\right]=\left[\kappa \mid a_{0} ; a_{1}, \ldots, a_{n-1}, i, 1\right]$, we obtain, in view of (ii),

$$
\delta_{n}(i, i+1) \leqslant \rho^{a_{1}+\cdots+a_{n-1}+i} / \rho^{2+2 a_{0}},
$$


whence

$$
\delta_{n}\left(a, a_{n}\right) \leqslant \sum_{i=a}^{a_{n}-1} \delta_{n}(i, i+1) \leqslant\left\{\sum_{j=0}^{\infty} \rho^{j-2}\right\} \frac{1}{\rho^{2 a_{0}}} \rho^{a_{1}+\cdots+a_{n-1}+a} .
$$

The inequality in (iii) follows from the fact that $\rho<1$.

Proof of Theorem 3.4. In view of Lemma 3.6, it suffices to establish the desired estimate of $\operatorname{Var}_{n}(\Phi)$. Fix $w \in \mathscr{J}^{n}$; if $w \notin\{\mathbf{0}, \mathbf{m}\}^{n}$, then

$$
\sup \left\{\left|\Phi(\xi)-\Phi\left(\xi^{\prime}\right)\right| ; \xi, \xi^{\prime} \in \llbracket w \rrbracket\right\}=0,
$$

so from here on we assume that $w \in\{\mathbf{0}, \mathbf{m}\}^{n}$. Let $\xi \in \llbracket w \rrbracket$ be of the form $\xi=w^{\prime} j \omega$, where

$$
w^{\prime}=\left\langle\kappa \star \mathbf{m} \mid a_{1}, \ldots, a_{k}\right\rangle
$$

(with $\kappa=\mathbf{0}$ or $\mathbf{1}$ ), $j \in \mathscr{J} \backslash\{\mathbf{0}, \mathbf{m}\}$ and $\omega \in \mathscr{J}^{\mathbf{N}}$ (the case of $\xi \in\{\mathbf{0}, \mathbf{m}\}^{\mathbf{N}}$ is handled in the same way). Then there exist $0<k_{n} \leqslant k$ and $0<a_{k_{n}}^{\prime} \leqslant a_{k_{n}}$ such that

$$
w=\xi_{0} \cdots \xi_{n-1}=\left\langle\kappa \star \mathbf{m} \mid a_{1}, \ldots, a_{k_{n}-1}, a_{k_{n}}^{\prime}\right\rangle
$$

(note that $n=a_{1}+\cdots+a_{k_{n}}^{\prime}$ ). Put

$$
\phi^{w}=\log \left(\Xi(\kappa \star \mathbf{m})\left[\hat{\kappa} \mid 1 ; a_{1}, \ldots, a_{k_{n}-1}, a_{k_{n}}^{\prime}\right]\right) .
$$

We have

$$
\Phi(\xi)-\phi^{w}=A_{k}+B_{k}, \quad \text { where }\left\{\begin{array}{l}
A_{k}:=\log \left(\frac{\left[\hat{\kappa}\left|1 ; a_{1}, \ldots, a_{k}\right| \Delta^{\hat{\kappa}+k} X_{j}\right]}{\left[\hat{\kappa} \mid 1 ; a_{1}, \ldots, a_{k_{n}-1}, a_{k_{n}}\right]}\right), \\
B_{k}:=\log \left(\frac{\left[\hat{\kappa} \mid 1 ; a_{1}, \ldots, a_{k_{n}-1}, a_{k_{n}}\right]}{\left[\hat{\kappa} \mid 1 ; a_{1}, \ldots, a_{k_{n}-1}, a_{k_{n}}^{\prime}\right]}\right)
\end{array}\right.
$$

(notice that $\phi^{w}=\Phi(w(\mathbf{m}+1) w(\mathbf{m}+1) \cdots)$ in the case $\mathbf{m} \geqslant 3$ ). Lemma 3.8 (iii) now yields

$$
\left|B_{k}\right| \leqslant K \rho^{a_{1}+\cdots+a_{k_{n}-1}+a_{k_{n}}^{\prime}} / \rho^{4}=K \rho^{n-4} .
$$

In order to establish a suitable upper bound of $\left|A_{k}\right|$ in (30), we first note that if $k>k_{n}$, then by definition,

$$
\left[\hat{\kappa}\left|1 ; a_{1}, \ldots, a_{k}\right| \Delta^{\hat{\kappa}+k} X_{j}\right]=\left[\hat{\kappa}\left|1 ; a_{1}, \ldots, a_{k_{n}}, a_{k_{n}+1}\right| Y\right],
$$

for some nonnegative column vector $Y$. Therefore, $\left[\hat{\kappa}\left|1 ; a_{1}, \ldots, a_{k}\right| \Delta^{\hat{\kappa}+k} X_{j}\right]$ lies between $\left[\hat{\kappa} \mid 1 ; a_{1}, \ldots, a_{k_{n}}\right]$ and $\left[\hat{\kappa} \mid 1 ; a_{1}, \ldots, a_{k_{n}}, a_{k_{n}+1}\right]$ and Lemma 3.8 (ii) yields

$$
\left|A_{k}\right| \leqslant \rho^{a_{1}+\cdots+a_{k_{n}}} / \rho^{6} \leqslant \rho^{n-6} .
$$

It remains to establish the upper bound for $\left|A_{k}\right|$ in the case $k=k_{n}$. Note that by Lemma 3.7,

$$
\left|A_{k}\right| \leqslant\left|\left[\hat{\kappa}\left|1 ; a_{1}, \ldots, a_{k}\right| \Delta^{\hat{\kappa}+k} X_{j}\right]-\left[\hat{\kappa} \mid 1 ; a_{1}, \ldots, a_{k}\right]\right| / \rho^{2} \leqslant \frac{v_{k} x_{2}}{u_{k} x_{1}+v_{k} x_{2}} \cdot \delta_{k} / \rho^{2},
$$


where $x_{1}$ and $x_{2}$ are the coordinates of $\Delta^{\hat{\kappa}+k} X_{j}$. Consider three different cases.

- The case $\alpha<1$. On one hand, if $\hat{\kappa}+k$ is odd, then

$$
\left|A_{k}\right| \leqslant \delta_{k} / \rho^{2} \leqslant \rho^{a_{1}+\cdots+a_{k}} / \rho^{6} .
$$

On the other hand, if $\hat{\kappa}+k$ is even, then there exists a constant $K^{\prime}>0$ such that

$$
\left|A_{k}\right| \leqslant \frac{v_{k} x_{2}}{u_{k} x_{1}} \delta_{k-1} / \rho^{2} \leqslant \frac{v_{k} x_{2}}{u_{k} x_{1}} \rho^{a_{1}+\cdots+a_{k-1}} / \rho^{6} \leqslant K^{\prime} \rho^{a_{1}+\cdots+a_{k}},
$$

because $v_{k} / u_{k} \leqslant \alpha^{a_{k}} / \alpha$ and $\left(\begin{array}{l}x_{1} \\ x_{2}\end{array}\right)=X_{j}$ is either $\left(\begin{array}{l}1 \\ \alpha\end{array}\right)$ or $\left(\begin{array}{l}1 \\ 0\end{array}\right)$. The claim now follows from (34), (35) and the fact that $\alpha^{\left(a_{1}+\cdots+a_{k}\right) / 2} \leqslant \rho^{n}$.

- The case $\alpha>1$ with $\mathbf{m}=2$. If $\hat{\kappa}+k$ is even, then

$$
\left|A_{k}\right| \leqslant \delta_{k} / \rho^{2} \leqslant \rho^{a_{1}+\cdots+a_{k}} / \rho^{6} .
$$

If $\hat{\kappa}+k$ is odd, then

$$
\left|A_{k}\right| \leqslant \frac{v_{k} x_{2}}{u_{k} x_{1}} \delta_{k-1} / \rho^{2} \leqslant \frac{v_{k}}{u_{k}} \cdot \frac{x_{2}}{x_{1}} \rho^{a_{1}+\cdots+a_{k}} / \rho^{6},
$$

as $v_{k} / u_{k} \leqslant \alpha^{-a_{k}} / \alpha^{-1}$ and $\left(\begin{array}{l}x_{1} \\ x_{2}\end{array}\right)=\Delta X_{j}$ is necessarily $\left(\begin{array}{c}\alpha \\ 1\end{array}\right)$ (because $\mathbf{m}=2$ ). The claim follows from (36), (37) and the fact that $\rho^{a_{1}+\cdots+a_{k}} \leqslant \rho^{n}$.

- The case $\alpha=1$. By (30),

$$
\begin{aligned}
\left|\Phi(\xi)-\phi^{w}\right| \leqslant & \left|\log \left(\frac{p_{k_{n}}}{q_{k_{n}}}\right)-\log \left(\frac{p_{k_{n}-1}}{q_{k_{n}-1}}\right)\right| \\
& +\mid\left\{\log \left(\frac{p_{k_{n}}}{q_{k_{n}}}\right)-\log \left(\frac{p_{k_{n}-1}}{q_{k_{n}-1}}\right)\right\} \\
& -\left\{\log \left(\frac{p_{k_{n}}^{\prime}}{q_{k_{n}}^{\prime}}\right)-\log \left(\frac{p_{k_{n}-1}}{q_{k_{n}-1}}\right)\right\} \mid,
\end{aligned}
$$

where $p_{k_{n}}^{\prime} / q_{k_{n}}^{\prime}$ denotes the continued fraction $\left[1 ; a_{1}, \ldots, a_{k_{n}-1}, a_{k_{n}}^{\prime}\right]$. Since these continued fractions are regular, it follows from the well known relations (see [15]) that

$$
\left|\Phi(\xi)-\phi^{w}\right| \leqslant \frac{2}{q_{k_{n}} q_{k_{n}-1}}+\frac{1}{q_{k_{n}}^{\prime} q_{k_{n}-1}} .
$$

By induction, $q_{k_{n}} \geqslant a_{1}+\cdots+a_{k_{n}}$ and $q_{k_{n}}^{\prime} \geqslant a_{1}+\cdots+a_{k_{n}-1}+a_{k_{n}}^{\prime}=n$, whence $\left|\Phi(\xi)-\phi^{w}\right| \leqslant 3 / n$.

\section{The Multifractal Analysis of the Measure $\mu$}

4.1. General case. The measure $\mu_{*}$ is $\mathfrak{F}^{-}$-weak Gibbs in all the cases described in Theorem 3.2, whence, in view of Theorem 1.4, the following claim holds:

Theorem 4.1. Let $\mu$ be the (p q)-distributed $(\beta, 2)$-Bernoulli convolution, where $\beta$ is the multinacci number of degree $\mathbf{m} \geqslant 2$, with an extra condition $\mathrm{p} \geqslant \mathrm{q}$ if $\mathbf{m} \geqslant 3$. The multifractal domain $\operatorname{DOM}\left(\mu_{*}\right)$ is a compact interval $[\underline{\alpha}, \bar{\alpha}]$, where

$$
-\infty<\underline{\alpha}:=\lim _{q \rightarrow+\infty} \frac{\tau_{\mu_{*}}(q)}{q} \leqslant \lim _{q \rightarrow-\infty} \frac{\tau_{\mu_{*}}(q)}{q}=: \bar{\alpha},
$$


and for any $\underline{\alpha} \leqslant \alpha \leqslant \bar{\alpha}$

$$
\operatorname{dim}_{H} E_{\mu_{*}}(\alpha)=\sup _{q \in \mathbf{R}}\left\{\alpha q-\tau_{\mu_{*}}(q)\right\} .
$$

We are now in position to state a multifractal formalism satisfied by the Bernoulli convolution $\mu$ itself.

Theorem 4.2. Assume $\mathrm{p} \geqslant \mathrm{q}$ whenever $\mathbf{m} \geqslant 3$; then $\underline{\alpha}<\alpha<\bar{\alpha}$ whenever $\operatorname{dim}_{H} E_{\mu}(\alpha)>0$, and for any $\underline{\alpha} \leqslant \alpha \leqslant \bar{\alpha}$,

$$
\operatorname{dim}_{H} E_{\mu}(\alpha)=\sup _{q \in \mathbf{R}}\left\{\alpha q-\tau_{\mu *}(q)\right\} .
$$

Remark 4.3. (1) We would like to emphasize that there exist cases when $\tau_{\mu}(q)$ is different from $\tau_{\mu_{*}}(q)$. This has to do with the fact that the multifractal formalism in Theorem 4.2 is not complete in the sense that $\operatorname{DOM}(\mu)$ may differ from the compact interval $[\underline{\alpha} ; \bar{\alpha}]=\operatorname{DoM}\left(\mu_{*}\right)$ (see Lemma 4.9).

(2) Let $\mu^{\prime}$ denote the (q p)-distributed $(\beta, 2)$-Bernoulli convolution. Here one has $\mu=\mu^{\prime} \circ \mathbb{S}$, where $\mathbb{S}$ is the symmetry: $\mathbb{S}(x)=\alpha_{\mu}-x$. This clearly implies that, for any $\alpha \in \mathbf{R}$

$$
\operatorname{dim}_{H} E_{\mu}(\alpha)=\operatorname{dim}_{H} E_{\mu^{\prime}}(\alpha) .
$$

Therefore, when $\mathrm{p}<\mathrm{q}$ and $\mathbf{m} \geqslant 3$, the multifractal formalism of $\mu$ is deduced by an application of Theorem 4.2 to the measure $\mu^{\prime}$.

It remains to prove Theorem 4.2; actually, it is a consequence of Theorem 4.1 and of the next proposition. Loosely speaking, the latter asserts that $\mu$ has a local Gibbs structure whenever $\mu_{*}$ has a global one (recall that the support of the measure $\mu$ is the interval $\left[0, \alpha_{\mu}\right]$ with $\left.\alpha_{\mu}=1 /(\beta-1)\right)$.

Proposition 4.4. For any $x$ in the support of $\mu$, there exists a constant $K_{x}$ such that

(i) for $r$ small enough, $\frac{1}{K_{x}} \leqslant \frac{\mu\left(B_{r}(x)\right)}{\mu_{*}\left(B_{r}(x)\right)} \leqslant K_{x}$, if $\left.x \in\right] 0,1[$;

(ii) for $r$ small enough, $\frac{1}{K_{x}} \leqslant \frac{\mu\left(B_{r}(x)\right)}{\mu_{*}\left(B_{r}\left(x-\dot{\mathrm{i}}_{2}\right)\right)} \leqslant K_{x}$, if $\left.x \in\right] 1 ; \alpha_{\mu}[$.

Proof. (i) Using the fact that $\mu$ and $\mu_{0}$ coincide on [0, 1[, we first compare their values on the $\mathfrak{F}$-cylinders different from $\llbracket \boldsymbol{0}^{k} \rrbracket$ for any $k \geqslant 1$. To do this, let $w=\mathbf{0}^{k} \eta w^{\prime}$, for $k \geqslant 0, \eta \neq \mathbf{0}$ and $w^{\prime}$ a (possibly empty) word in $\mathscr{J}^{*}$; since $\left(\begin{array}{ll}1 & 0\end{array}\right) P_{0}=\mathrm{p}^{\mathbf{m}}\left(\begin{array}{ll}1 & 0\end{array}\right)$ and $\left(\begin{array}{ll}1 & 1\end{array}\right) P_{0} \leqslant\left(\begin{array}{ll}1 & 1\end{array}\right)$, we have

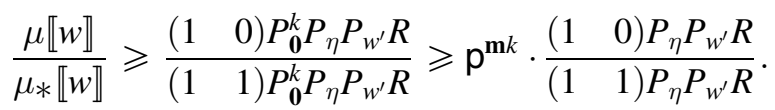

Moreover, $\left(\begin{array}{ll}1 & 0\end{array}\right) P_{\eta} \geqslant \mathrm{p}^{\mathbf{m}} \mathrm{q}^{\mathbf{m}}\left(\begin{array}{ll}1 & 1\end{array}\right)$ and $\left(\begin{array}{ll}1 & 1\end{array}\right) P_{\eta} \leqslant\left(\begin{array}{ll}1 & 1\end{array}\right)$, whence

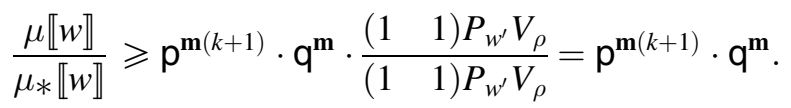

Since the upper bound $\mu \llbracket w \rrbracket / \mu_{*} \llbracket w \rrbracket \leqslant 2$ is always valid, we obtain

$$
C^{k+1} \leqslant \frac{\mu \llbracket w \rrbracket}{\mu_{*} \llbracket w \rrbracket} \leqslant 2,
$$


where $C=(\mathrm{pq})^{\mathbf{m}}$. Let $0<x<1$ and let $k_{x} \geqslant 1$ be an integer such that $x \notin \llbracket \mathbf{0}^{k_{x}} \rrbracket$; any ball $\left.B_{r}(x) \subset\right] 0,1\left[\right.$ which does not intersect $\llbracket \mathbf{0}^{k_{x}+1} \rrbracket$ can be tiled by countably many cylinders of the form $\llbracket 0^{k} \eta w^{\prime} \rrbracket$, where $k \leqslant k_{x}, w \neq 0$ and $w^{\prime} \in \mathscr{J}^{*}$. Now it follows from (38) that

$$
C^{k_{x}+1} \leqslant \frac{\mu\left(B_{r}(x)\right)}{\mu_{*}\left(B_{r}(x)\right)} \leqslant 2 .
$$

(ii) Now, let $x \in] 1, \alpha_{\mu}\left[\right.$. The Bernoulli convolutions $\mu$ and $\mu^{\prime}$ associated with the probability vectors $(\mathrm{p}, \mathrm{q})$ and $(\mathrm{q}, \mathrm{p})$ respectively, satisfy the relation $\mu=\mu^{\prime} \circ \mathbb{S}$, where $\mathbb{S}(t)=\alpha_{\mu}-t$ for any $t \in\left[0, \alpha_{\mu}\right]$. Since $x^{\prime}=\mathbb{S}(x)$ belongs to ] $0,1\left[\right.$, there exists $k_{x} \geqslant 1$ such that $x^{\prime} \notin \llbracket \mathbf{0}^{k_{x}} \rrbracket$; we apply (39) to the measures $\mu^{\prime}$ and $\mu_{*}^{\prime}$ at $x^{\prime}$ and obtain

$$
C^{k_{x}+1} \leqslant \frac{\mu^{\prime}\left(B_{r}\left(x^{\prime}\right)\right)}{\mu_{*}^{\prime}\left(B_{r}\left(x^{\prime}\right)\right)} \leqslant 2 .
$$

This yields (ii), as $\mu^{\prime}\left(B_{r}\left(x^{\prime}\right)\right)=\mu\left(B_{r}(x)\right)$, and

$$
\mu_{*}^{\prime}\left(B_{r}\left(x^{\prime}\right)\right)=\frac{\mu^{\prime}\left(B_{r}\left(x^{\prime}\right)\right)+\mu^{\prime}\left(B_{r}\left(x^{\prime}+\dot{i}_{2}\right)\right)}{\mu^{\prime}([0 ; 1])+\mu^{\prime}\left([0 ; 1]+\dot{i}_{2}\right)}=\lambda \mu_{*}\left(B_{r}\left(x-\dot{i}_{2}\right)\right),
$$

where $\lambda>0$ is a constant.

4.2. Case of the Erdős measure. As we have seen, "local" Gibbs properties of the Bernoulli convolution in a multinacci base are sufficient to establish the multifractal formalism of the level sets with positive Hausdorff dimension. However, a natural question would be to determine whether or not there exists a reasonable net with respect to which the measure in question has a "global" Gibbs structure.

In the rest of the paper we concentrate on the case of the Erdös measure, i.e. $\mathbf{m}=2$ or, equivalently, $\beta=(1+\sqrt{5}) / 2$. Following our notation introduced in Section 3.1, the $\beta$-shift is associated with the two affine contractions:

$$
\mathbb{R}_{0}(x)=x / \beta \quad \text { and } \quad \mathbb{R}_{1}(x)=x / \beta+1 / \beta .
$$

The corresponding s.a.c. $\mathscr{R}$ consists of the three contractions:

$$
\begin{aligned}
& \hat{\mathbb{R}}_{\mathbf{0}}(x)=\mathbb{R}_{00}(x)=x / \beta^{2}, \quad \hat{\mathbb{R}}_{\mathbf{1}}(x)=\mathbb{R}_{010}(x)=x / \beta^{3}+1 / \beta^{2}, \\
& \hat{\mathbb{R}}_{\mathbf{2}}(x)=\mathbb{R}_{10}(x)=x / \beta^{2}+1 / \beta .
\end{aligned}
$$

It is adapted to the interval $[0,1]$ (see Fig. 4). Recall that the measure $\mu$ is supported by the interval $[0, \beta]$; to study the global Gibbs properties of $\mu$, it is thus more convenient to make an affine scale change from $[0,1]$ to $[0, \beta]$. Thus, instead of $\mathscr{R}$ we consider the s.a.c. $\mathscr{S}=\left\{\mathbb{S}_{\mathbf{0}}, \mathbb{S}_{\mathbf{1}}, \mathbb{S}_{\mathbf{2}}\right\}$ with

$$
\mathbb{S}_{\mathbf{0}}(x)=x / \beta^{2}, \quad \mathbb{S}_{\mathbf{1}}(x)=x / \beta^{3}+1 / \beta, \quad \mathbb{S}_{\mathbf{2}}(x)=x / \beta^{2}+1 .
$$

Clearly, $\mathscr{S}$ is adapted to the interval $[0 ; \beta]$ and we denote the associated 3 -fold net by $\tilde{\mathfrak{F}}$. For any word $w \in\{\mathbf{0}, \mathbf{1}, \mathbf{2}\}^{*}$, we put $\llbracket w \rrbracket=\mathbb{S}_{w}[0, \beta[$; obviously, the set of $\llbracket w \rrbracket$ determines the basic intervals of $\tilde{\mathfrak{F}}$. Moreover, any Borel measure $\nu$ on the real line is associated with the measure $\tilde{\nu}$ defined on an arbitrary interval $J$ as 
follows: $\tilde{\nu}(J)=\nu(J / \beta)$. This scale change clearly implies that the measures $\tilde{\mu}_{0}, \tilde{\mu}_{2}$ and $\tilde{\mu}_{*}$ satisfy, for any word $w \in\{\mathbf{0}, \mathbf{1}, \mathbf{2}\}^{*}$, the following matrix identities:

$$
\left(\begin{array}{c}
\tilde{\mu}_{0} \llbracket w \rrbracket \\
\tilde{\mu}_{2} \llbracket w \rrbracket
\end{array}\right)=P_{w}\left(\begin{array}{c}
\mu_{0}([0,1]) \\
\mu_{2}([0,1])
\end{array}\right) \quad \text { and } \quad \tilde{\mu}_{*} \llbracket w \rrbracket=\left(\begin{array}{ll}
1 & 1
\end{array}\right) P_{w}\left(\begin{array}{c}
\mathrm{p} \\
\mathrm{q}
\end{array}\right),
$$

where

$$
P_{0}=\mathrm{p}^{2}\left(\begin{array}{cc}
1 & 0 \\
\mathrm{q} / \mathrm{p} & \mathrm{q} / \mathrm{p}
\end{array}\right), \quad P_{1}=\mathrm{p}^{2} \mathrm{q}\left(\begin{array}{cc}
1 & 1 \\
\mathrm{q} / \mathrm{p} & \mathrm{q} / \mathrm{p}
\end{array}\right), \quad P_{2}=\mathrm{q}^{2}\left(\begin{array}{cc}
\mathrm{p} / \mathrm{q} & \mathrm{p} / \mathrm{q} \\
0 & 1
\end{array}\right) \text {. }
$$

We are going to use two key properties of this model. Firstly, the probability measure $\mu$ satisfies the following well known self-similar equation:

$$
\mu=\mathrm{p} \mu \circ \mathbb{R}_{0}^{-1}+\mathrm{q} \mu \circ \mathbb{R}_{1}^{-1} .
$$

Secondly,

$$
\mathbb{S}_{\mathbf{0}}=\mathbb{R}_{00}, \quad \mathbb{S}_{1}=\mathbb{R}_{100}=\mathbb{R}_{011}, \quad \mathbb{S}_{\mathbf{2}}=\mathbb{R}_{11},
$$

where the identity $\mathbb{R}_{100}=\mathbb{R}_{011}$ plays a crucial role. Let $J$ be a subinterval of $[0, \beta]$; then, one has successively

$$
\begin{aligned}
-\mu\left(\mathbb{S}_{\mathbf{0}}(J)\right) & =\mathrm{p} \mu\left(\mathbb{R}_{0}^{-1} \mathbb{R}_{00}(J)\right)+\mathrm{q} \mu\left(\mathbb{R}_{1}^{-1} \mathbb{R}_{00}(J)\right) \\
& =\mathrm{p} \mu\left(\mathbb{R}_{0}(J)\right)=\mathrm{p} \tilde{\mu}_{0}(J) \\
\text { - } \mu\left(\mathbb{S}_{\mathbf{1}}(J)\right) & =\mathrm{p} \mu\left(\mathbb{R}_{0}^{-1} \mathbb{R}_{011}(J)\right)+\mathrm{q} \mu\left(\mathbb{R}_{1}^{-1} \mathbb{R}_{100}(J)\right) \\
& =\mathrm{p} \mu\left(\mathbb{R}_{11}(J)\right)+\mathrm{q} \mu\left(\mathbb{R}_{00}(J)\right) \\
& =\mathrm{pq} \mu\left(\mathbb{R}_{1}(J)\right)+\mathrm{qp} \mu\left(\mathbb{R}_{0}(J)\right)=\mathrm{pq}\left(\tilde{\mu}_{0}(J)+\tilde{\mu}_{2}(J)\right) \\
\text { - } \mu\left(\mathbb{S}_{\mathbf{2}}(J)\right) & =\mathrm{p} \mu\left(\mathbb{R}_{0}^{-1} \mathbb{R}_{11}(J)\right)+\mathrm{q} \mu\left(\mathbb{R}_{1}^{-1} \mathbb{R}_{11}(J)\right) \\
& =\mathrm{q} \mu\left(\mathbb{R}_{1}(J)\right)=\mathrm{q} \tilde{\mu}_{2}(J) .
\end{aligned}
$$

We conclude by the fact that, for any $\eta \in\{\mathbf{0}, \mathbf{1}, \mathbf{2}\}$ and any $w \in\{\mathbf{0}, \mathbf{1}, \mathbf{2}\}^{*}$, one has

$$
\mu \llbracket \eta w \rrbracket=V_{\eta} P_{w}\left(\begin{array}{c}
\mathrm{p} /(1-\mathrm{pq}) \\
\mathrm{q} /(1-\mathrm{pq})
\end{array}\right), \quad \text { where }\left\{\begin{array}{l}
V_{0}=\left(\begin{array}{ll}
\mathrm{p} & 0
\end{array}\right) ; \\
V_{1}=(\mathrm{pq} \\
V_{2}=\left(\begin{array}{ll}
0 & \mathrm{q}
\end{array}\right) .
\end{array}\right.
$$

Consider two subcases.

4.2.1. The uniform case. We first consider the uniform Erdős measure, i.e., $\mathrm{p}=\mathrm{q}=1 / 2$. Then for $\eta \in\{\mathbf{0}, \mathbf{1}, \mathbf{2}\}$ and $w \in\{\mathbf{0}, \mathbf{1}, \mathbf{2}\}^{*}$ :

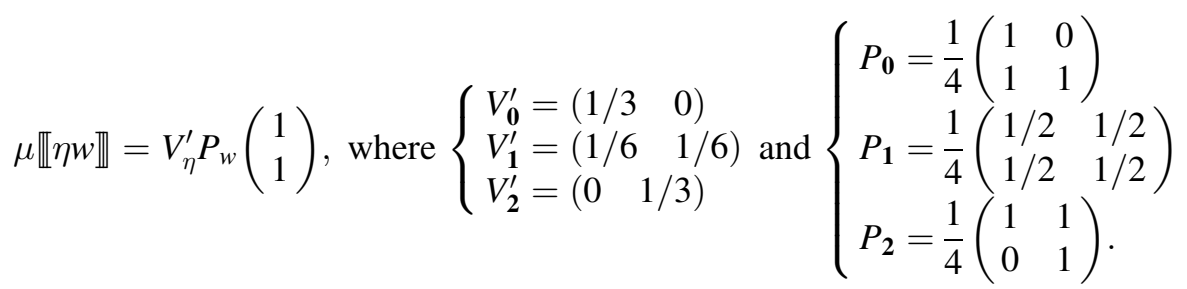


We are going to show that $\mu$ is $\tilde{\widetilde{F}}$-weak Gibbs. Consider the probability measure $\tilde{\mu}_{*}$ with the support equal to the interval $[0, \beta]$ defined as follows: for any word $w \in\{\mathbf{0}, \mathbf{1}, \mathbf{2}\}^{*}$,

$$
\tilde{\mu}_{*} \llbracket w \rrbracket=\frac{1}{2}\left(\begin{array}{ll}
1 & 1
\end{array}\right) P_{w}\left(\begin{array}{l}
1 \\
1
\end{array}\right) .
$$

By Theorem 3.4, $\tilde{\mu}_{*}$ is a $\tilde{\widetilde{F}}$-weak Gibbs measure with the potential $\Phi:\{\mathbf{0}, \mathbf{1}, \mathbf{2}\}^{\mathbf{N}} \rightarrow \mathbf{R}$; the formula for $\Phi$ can in fact be given by means of regular continued fractions: let $a_{0}, \ldots, a_{2 n}$ be $2 n$ integers $(n \geqslant 0)$ with $a_{0}, \ldots, a_{2 n-1}>0$ and $a_{2 n} \geqslant 0$, when $n \geqslant 1$. Then for any $\xi \in\{\mathbf{0}, \mathbf{1}, \mathbf{2}\}^{\mathbf{N}}$,

$$
\begin{aligned}
\Phi\left(\mathbf{0}^{a_{0}} \mathbf{2}^{a_{1}} \cdots \mathbf{2}^{a_{2 n-1}} \mathbf{0}^{a_{2 n}} \mathbf{1} \xi\right) & =\Phi\left(\mathbf{2}^{a_{0}} \mathbf{0}^{a_{1}} \ldots \mathbf{0}^{a_{2 n-1}} \mathbf{2}^{a_{2 n}} \mathbf{1} \xi\right) \\
& =\log \left(f\left(a_{0}, \ldots, a_{2 n}\right) / 4\right)
\end{aligned}
$$

with $f(0)=1$ and

$$
f\left(a_{0}, \ldots, a_{2 n}\right)=1+\frac{1}{a_{0}+\frac{1}{\ddots \quad+\frac{1}{a_{2 n}+1}} .}
$$

Theorem 4.5. The uniform Erdös measure $\mu$ is a $\tilde{\widetilde{F}}$-weak Gibbs measure of the potential $\Phi$.

This theorem is a consequence of the fact that $\tilde{\mu}_{*}$ is itself a $\tilde{\tilde{F}}$-weak Gibbs measure of $\Phi$ and of the following proposition:

Proposition 4.6. For any $\omega \in\{\mathbf{0}, \mathbf{1}, \mathbf{2}\}^{\mathbf{N}}$ and any integer $n \geqslant 1$,

$$
\frac{8}{3(n+2)} \leqslant \frac{\mu \llbracket \omega_{0} \cdots \omega_{n-1} \rrbracket}{\tilde{\mu}_{*} \llbracket \omega_{0} \cdots \omega_{n-1} \rrbracket} \leqslant \frac{8}{3} .
$$

Proof. Note first that $\mu \llbracket w \rrbracket / \tilde{\mu}_{*} \llbracket w \rrbracket=4 / 3$, whenever $w=\mathbf{1} w^{\prime}$; moreover, if $w=\mathbf{0}^{n}$ or $\mathbf{2}^{n}$ with $n \geqslant 1$, then $\mu \llbracket w \rrbracket / \tilde{\mu}_{*} \llbracket w \rrbracket=8 /(3(n+2))$. Now, given $n \geqslant 2$, we assume that $w=\eta^{a} \nu w^{\prime} \in\{\mathbf{0}, \mathbf{1}, \mathbf{2}\}^{n}$, with $a<n$. Without loss of generality we assume $\eta=\mathbf{0}$ and $\nu=\mathbf{2}$ (the other cases with $\eta \in\{\mathbf{0}, \mathbf{2}\}$ and $\eta \neq \nu \in\{\mathbf{0}, \mathbf{1}, \mathbf{2}\}$ are similar). It is straightforward that $\mu \llbracket w \rrbracket / \tilde{\mu}_{*} \llbracket w \rrbracket \leqslant 8 / 3$, so it remains to establish the lower bound of $\mu \llbracket w \rrbracket / \tilde{\mu}_{*} \llbracket w \rrbracket$. As

$$
P_{w^{\prime}}\left(\begin{array}{l}
1 \\
1
\end{array}\right):=\left(\begin{array}{l}
x \\
y
\end{array}\right)
$$

we have

$$
\frac{\mu \llbracket w \rrbracket}{\tilde{\mu}_{*} \llbracket w \rrbracket}=\frac{8}{3 \cdot 4^{a+1}} \cdot \frac{\left(\begin{array}{ll}
1 & 1
\end{array}\right)\left(\begin{array}{l}
x \\
y
\end{array}\right)}{\left(\begin{array}{ll}
1 & 1
\end{array}\right) P_{0}^{a} P_{2}\left(\begin{array}{l}
x \\
y
\end{array}\right)}=\frac{8}{3} \cdot \frac{(x+y)}{(1+a)(x+y)+y} \geqslant \frac{8}{3(a+2)} .
$$

Since $a<n$, we conclude that $\mu \llbracket w \rrbracket / \tilde{\mu}_{*} \llbracket w \rrbracket \geqslant \frac{8}{3(n+2)}$. 
We would like to stress that $\mu$ is not a $\tilde{\mathbb{F}}$-Gibbs measure. Actually, if $\overline{0}:=\left(\omega_{i}=0\right)_{i=0}^{\infty}$ then, for any potential $\psi: \Sigma \rightarrow \mathbf{R}$, we have

$$
\exp \left(S_{n} \psi(\mathbf{2} \overline{\mathbf{0}})\right)=\frac{\exp (\psi(\mathbf{2} \overline{\mathbf{0}}))}{\exp (\psi(\overline{\mathbf{0}}))}\{\exp (\psi(\overline{\mathbf{0}}))\}^{n} .
$$

A direct computation, in view of (41), yields $\mu \llbracket \mathbf{2 0}^{n-1} \rrbracket=n /\left(3 \cdot 4^{n-1}\right)$, for any $n>0$; if $\mu$ were a Gibbs measure of $\psi$, then there would exist a constant $K>1$ such that for any $n \geqslant 1$,

$$
\frac{1}{K} \leqslant \frac{1}{n} \cdot\{4 \cdot \exp (\psi(\overline{\mathbf{0}}))\}^{n} \leqslant K,
$$

which is impossible. Hence $\mu$ is not $\tilde{\widetilde{F}}$-Gibbs.

Furthermore, in this specific case one can prove a much more refined result, namely that $\mu$ is not Gibbs in a very strong sense - see Theorem 4.7 below.

Assume that $\mathfrak{E}$ is an arbitrary $\mathbf{s}$-fold net which is quasi-Bernoulli; if $\mu$ is $\mathfrak{E}$ Gibbs then, in particular, it is $\mathfrak{E}$-quasi-Bernoulli and by Theorem 1.3 (i), its scale spectrum $\tau_{\mu}$ is differentiable on the whole real line. However, this is not the case, since it is known [6,7] that there exists $q_{c}<-2$ such that $\tau_{\mu}$ is not differentiable at $q_{c}$.

Thus, we have proved.

Theorem 4.7. There is no quasi-Bernoulli net with respect to which the uniform Erdös measure is quasi-Bernoulli.

4.2.2. The nonuniform case. From now on we assume $\mathrm{p} \neq \mathrm{q}$. As the measure $\tilde{\mu}_{*}$ is $\tilde{\mathfrak{F}}$-Gibbs, its multifractal domain is a compact interval. In fact,

$$
\operatorname{Dom}\left(\mu_{*}\right)=\operatorname{Dom}\left(\tilde{\mu}_{*}\right):=[\underline{\alpha}, \bar{\alpha}],
$$

where

$$
\underline{\alpha}=\inf _{x \in[0, \beta]}\left\{\lim _{r \rightarrow 0} \frac{\log \tilde{\mu}_{*}\left(B_{r}(x)\right)}{\log (r)}\right\}=\inf _{\omega \in\{\mathbf{0 , 1 , 2}\}^{\mathrm{N}}}\left\{\lim _{n \rightarrow \infty} \frac{\log \tilde{\mu}_{*} \llbracket \omega_{0} \cdots \omega_{n-1} \rrbracket}{\log \left|\llbracket \omega_{0} \cdots \omega_{n-1} \rrbracket\right|}\right\}
$$

and

$$
\bar{\alpha}=\sup _{x \in[0, \beta]}\left\{\lim _{r \rightarrow 0} \frac{\log \tilde{\mu}_{*}\left(B_{r}(x)\right)}{\log (r)}\right\}=\sup _{\omega \in\{\mathbf{0 , 1}, \mathbf{2}\}^{\mathrm{N}}}\left\{\lim _{n \rightarrow \infty} \frac{\log \tilde{\mu}_{*} \llbracket \omega_{0} \cdots \omega_{n-1} \rrbracket}{\log \left|\llbracket \omega_{0} \cdots \omega_{n-1} \rrbracket\right|}\right\} .
$$

Theorem 4.8. Assume that $\mathrm{p} \neq \mathrm{q}$; then there is no quasi-Bernoulli net with respect to which the corresponding (nonuniform) Erdös measure is quasi-Bernoulli or weak Gibbs.

If $\mu$ were a weak Gibbs measure w.r.t. a quasi-Bernoulli net of the interval $[0, \beta]$, it would be necessary that its multifractal domain were a compact interval. Moreover, by Proposition 4.4, one would have

$$
\operatorname{Dom}(\mu)=\operatorname{Dom}\left(\mu_{*}\right)=\operatorname{Dom}\left(\tilde{\mu}_{*}\right)=[\underline{\alpha}, \bar{\alpha}] .
$$

Thus, Theorem 4.8 would follow from 
Lemma 4.9. If $\mathrm{p} \neq \mathrm{q}$ then

$$
\operatorname{Dom}(\mu) \neq \operatorname{Dom}\left(\mu_{*}\right)=\operatorname{Dom}\left(\tilde{\mu}_{*}\right)=[\underline{\alpha}, \bar{\alpha}] .
$$

Proof. Assume without loss of generality that $p<q-$ the case $p>q$ is handled by considering the symmetry between the $(p q)$ and the $(q p)$-distributed Erdös measure. On one hand, given any $\omega \in\{\mathbf{0}, \mathbf{1}, \mathbf{2}\}^{\mathbf{N}}$, as is easy too see, there exists a constant $K>0$ such that

$$
\tilde{\mu}_{*} \llbracket \omega_{0} \cdots \omega_{n-1} \rrbracket \geqslant K(\mathrm{pq})^{n}
$$

so that

$$
\frac{\log \tilde{\mu}_{*} \llbracket \omega_{0} \cdots \omega_{n-1} \rrbracket}{\log \left|\llbracket \omega_{0} \cdots \omega_{n-1} \rrbracket\right|} \leqslant \frac{\log K+n \log (\mathrm{pq})}{2 n \log (1 / \beta)},
$$

whence

$$
\bar{\alpha} \leqslant \frac{\log (\mathrm{pq})}{2 \log (1 / \beta)} .
$$

On the other hand, a direct computation yields that for any integer $n \geqslant 0$,

$$
\mu \llbracket \boldsymbol{0}^{n} \rrbracket=\mathrm{p}^{2 n-2}(\mathrm{p} \quad 0)\left(\begin{array}{cc}
1 & 0 \\
\mathrm{q} / \mathrm{p} & \mathrm{q} / \mathrm{p}
\end{array}\right)^{n-1}\left(\begin{array}{l}
\mathrm{p} /(1-\mathrm{pq}) \\
\mathrm{q} /(1-\mathrm{pq})
\end{array}\right)=K^{\prime} \mathrm{p}^{2 n}
$$

so that

$$
\lim _{r \rightarrow 0} \frac{\log \mu \llbracket \mathbf{0}^{n} \rrbracket}{\log \left|\llbracket \mathbf{0}^{n} \rrbracket\right|}=\frac{\log \mathrm{p}}{\log (1 / \beta)}
$$

Given any $0<r<\beta$, let $n_{r}$ be the integer satisfying $1 / \beta^{2\left(n_{r}+1\right)} \leqslant r<1 / \beta^{2 n_{r}}$, whence

$$
\begin{aligned}
\frac{n_{r} \log \left(1 / \beta^{2}\right)}{\log r} \cdot \frac{\log \mu \llbracket \mathbf{0}^{n_{r}} \rrbracket}{\log \left|\llbracket \mathbf{0}^{n_{r}} \rrbracket\right|} & \leqslant \frac{\log \mu\left(B_{r}(0)\right)}{\log r} \\
& \leqslant \frac{\left(n_{r}+1\right) \log \left(1 / \beta^{2}\right)}{\log r} \cdot \frac{\log \mu \llbracket \mathbf{0}^{\left(n_{r}+1\right)} \rrbracket}{\log \left|\llbracket \mathbf{0}^{\left(n_{r}+1\right)} \rrbracket\right|} .
\end{aligned}
$$

Since $p<q$, we have, in view of (43) and (44),

$$
\lim _{r \rightarrow 0} \frac{\log \mu\left(B_{r}(0)\right)}{\log r}=\frac{\log \mathrm{p}}{\log (1 / \beta)}>\frac{\log (\mathrm{pq})}{2 \log (1 / \beta)} \geqslant \bar{\alpha} .
$$

Remark 4.10. One can prove that the multifractal domains of $\mu$ and $\mu *$ differ because of the local dimension of $\mu$ at the point $x=0$ if $\mathrm{p}<\mathrm{q}$ and $x=\alpha_{\mu}$ if $\mathrm{p}>\mathrm{q}$. Therefore, the multifractal domain of the nonuniform Erdös measure is the disconnected union of an interval and a singleton. More precisely,

$$
\operatorname{Dom}(\mu)=[\underline{\alpha}, \bar{\alpha}] \cup\left\{\alpha^{*}\right\} \quad \text { with } \alpha^{*}=\max \left\{\frac{\log p}{\log (1 / \beta)}, \frac{\log \mathrm{q}}{\log (1 / \beta)}\right\}
$$


A similar pattern holds in the case of the 3-fold convolution of the Cantor measure. Namely, let $\gamma$ denote the Cantor measure, i.e., the self-similar probability measure associated with the two affine contractions

$$
\mathbb{S}_{\mathbf{0}}: x \mapsto x / 3 \text { and } \mathbb{S}_{1}: x \mapsto x / 3+2 / 3 .
$$

It can be easily checked that the 3-fold convolution measure $\gamma^{\prime}:=\gamma * \gamma * \gamma$ is none other than the p-distributed $(2,3)$-Bernoulli convolution with

$$
\mathrm{p}=\left(\begin{array}{llll}
\frac{1}{8} & \frac{3}{8} & \frac{3}{8} & \frac{1}{8}
\end{array}\right)
$$

A detailed multifractal analysis of $\gamma^{\prime}$ presented in [11] shows that the multifractal domain $\operatorname{Dom}\left(\gamma^{\prime}\right)$ is no longer a compact interval. Actually, $\operatorname{DoM}\left(\gamma^{\prime}\right)$ is proved to be the union of a compact interval and a singleton. This proves that $\gamma^{\prime}$ is neither quasi-Bernoulli nor weak Gibbs w.r.t. any given reasonable s-fold net of the unit interval.

Acknowledgment. A part of this paper was written during the postdoctoral visit of the first author at the Department of Mathematics of the Chinese University of Hong Kong. He is grateful to Jean-Pierre Kahane and Ka-Sing Lau for their constant support and valuable discussions.

\section{References}

[1] Alexander J, Yorke J (1984) Fat baker's transformations. Ergod Theory Dynam Systems 4: $1-23$

[2] Borwein P, Hare KG (2001) Some computations on the spectra of Pisot and Salem numbers. Math Comp 71: 767-780

[3] Bowen R (1975) Equilibrium states and the Ergodic Theory of Anosov Diffeomorphisms. Lect Notes Math 470. Berlin Heidelberg New York: Springer

[4] Brown G, Michon G, Peyrière J (1992) On the multifractal analysis of measures. J Stat Phys 6: 775-790

[5] Erdős P (1939) On a family of symmetric Bernoulli convolutions. Amer J Math 61: 974-976

[6] Feng D-J (2005) The limit Rademacher functions and Bernoulli convolutions associated with Pisot numbers. Adv Math (to appear)

[7] Feng D-J, Olivier E (2003) Multifractal analysis of weak Gibbs measures and phase transition application to some Bernoulli convolutions. Ergod Theory Dynam Systems 23: 1751-1784

[8] Garsia AM (1963) Entropy and singularity of infinite convolutions. Pac J Math 13: 1159-1169

[9] Heurteaux Y (1998) Estimation de la dimension inférieure et de la dimension supérieure des mesures. Ann Inst Henri Poincaré 34: 309-338

[10] Hofbauer F (1995) Local dimension for piecewise monotonic maps on the interval. Ergod Theory Dynam Systems 15: 1119-1142

[11] Hu T-Y, Lau K-S (2001) Multifractal structure of convolution of the Cantor measure. Adv Appl Math 27: 1-16

[12] Jessen B, Wintner A (1935) Distribution functions and the Riemann zeta function. Trans Amer Math Soc 38: 48-88

[13] Kahane JP, Salem R (1958) Sur la convolution d'une infinité de distributions de Bernoulli. Colloq Math VI: $193-202$

[14] Keane M, Smorodinsky M, Solomyak B (1995) On the morphology of $\gamma$-expansion with deleted digits. Trans Amer Math Soc 347: 955-966

[15] Khintchin A (1964) Continued Fractions. Chicago: Univ Press

[16] Lalley SP (1998) Random series in powers of algebraic integers, Hausdorff dimension of the limit distribution. J Lond Math Soc II. Ser 57: 629-654

[17] Lau K-S (1993) Dimension of a family of singular Bernoulli convolutions. J Funct Anal 116: $335-358$

[18] Lau K-S, Ngai S-M (1998) $L^{q}$-spectrum of the Bernoulli convolution associated with the Golden Ratio. Studia Math 131: 225-251 
[19] Ledrappier F, Porzio A (1994) A Dimension formula for Bernoulli convolution. J Stat Phys 76: $1307-1327$

[20] Olivier E, Thomas A (2004) Infinite product of $2 \times 2$ matrices and the Gibbs properties of Bernoulli convolutions in $\beta$-numeration. Preprint

[21] Olivier E, Thomas A (2004) On the Gibbs properties of Bernoulli convolutions related to the numeration in integral base. Preprint

[22] Olsen L (2000) Mulifractal geometry. In: Bandt C et al. (eds) Fractal Geometry and Stochastics, II (Proceedings Greif-swald/Koserow, 1998), pp 3-37. Basel: Birkhäuser

[23] Peres Y, Schlag W, Solomyak B (2000) Sixty years of Bernoulli convolutions, II (Greifswald/ Koserow, 1998), pp 39-65, Basel: Birkhäuser

[24] Perron O (1950) Die Lehre von den Kettenbrüchen, 2nd ed (German). New York: Chelsea

[25] Pesin Y (1997) Dimension Theory in Dynamical Systems. Chicago: Univ Press

[26] Pesin Y, Weiss H (1997) The multifractal analysis of Gibbs measures: Motivation, mathematical foundation, and examples. Chaos 7: 89-106

[27] Pollicott M, Simon K (1995) The Hausdorff dimension of $\lambda$-expansions with deleted digits. Trans Amer Math Soc 347: 967-983

[28] Rand DA (1989) The singularity spectrum $f(\alpha)$ for cookie-cutters. Ergod Theory Dynam Systems 9: $527-541$

[29] Sidorov N (2003) Ergodic-theoretic properties of certain Bernoulli convolutions. Acta Math Hungar 101: $345-355$

[30] Sidorov N, Vershik A (1998) Ergodic properties of the Erdős measure, the entropy of the goldenshift, and related problems. Monatsh Math 126: 215-261

[31] Solomyak B (1994) Conjugates of beta-numbers and the zero-free domain for a class of analytic functions. Proc London Math Soc 68: 477-498

[32] Yuri M (1998) Zeta functions for certain non-hyperbolic systems and topological Markov approximations. Ergod Theory Dynam Systems 18: 1589-1612

Authors' addresses: Eric Olivier, SCAM, Université de Provence, 3, place Victor Hugo, 13331 Marseille Cedex 3, France; e-mail: eric.olivier@up.univ-mrs.fr; Nikita Sidorov, School of Mathematics, The University of Manchester, P.O. Box 88, Manchester M60 1QD, United Kingdom; e-mail: sidorov@ manchester.ac.uk; Alain Thomas, Centre de Mathématiques et d'Informatique, LATP Équipe DSA, 39, rue F. Joliot-Curie, 13453 Marseille Cedex 13, France; e-mail: thomas@gyptis.univ-mrs.fr 